\title{
Large time asymptotics for partially dissipative hyperbolic systems
}

\author{
K. BeAuchard ${ }^{*}$ E. ZuazuA ${ }^{\dagger \ddagger}$
}

\begin{abstract}
This work is concerned with ( $n$-component) hyperbolic systems of balance laws in $m$ space dimensions. First we consider linear systems with constant coefficients and analyze the possible behavior of solutions as $t \rightarrow \infty$. Using Fourier transform we exhibit the role that control theoretical tools, such as the classical Kalman rank condition, play. We build Lyapunov functionals allowing to establish explicit decay rates depending on the frequency variable. In this way we extend the previous analysis by Shizuta and Kawashima under the so-called algebraic condition (SK). In particular we show the existence of systems exhibiting a more complex behavior than the one that the (SK) condition allows. We also discuss the link of this analysis with previous literature in the context of damped wave equations, hypoellipticity and hypocoercivity. To conclude we analyze the existence of global solutions around constant equilibria for nonlinear systems of balance laws. Our analysis of the linear case allows proving existence results in situations that the previously existing theory does not cover.
\end{abstract}

\section{Contents}

1 Introduction and main results 2

1.1 Problem formulation ................ . . . 2

1.2 Main results . . . . . . . . . . . . . . . . . 6

2 Rank conditions, (SK), hypocoercivity and decay rates 9

2.1 (SK) and Kalman rank conditions . . . . . . . . . . . . 9

2.2 Lyapunov functionals and explicit decay rates . . . . . . . . . . . 10

2.3 A new proof for Shizuta and Kawashima's decomposition . . . . 14

*CMLA, ENS Cachan, CNRS, Universud, 61 avenue du Président Wilson, F-94230 Cachan, France, email: Karine.Beauchard@cmla.ens-cachan.fr

†IMDEA-Matemáticas \& Departamento de Matemáticas, Facultad de Ciencias, Universidad Autónoma, Cantoblanco, 28049 Madrid, Spain email: enrique.zuazua@uam.es

¥The work of the second author has been supported by the Grant MTM2005-00714, the DOMINO Project CIT-370200-2005-10 in the PROFIT program and the Ingenio Mathematica (i-MATH) project of the Program CONSOLIDER INGENIO 2010 of the MEC (Spain) and the SIMUMAT project of the CAM (Spain). This work started while the first author was visiting the Universidad Autónoma de Madrid as a postdoc within the SIMUMAT project and it finished while the second author was visiting the Isaac Newton Instiute in Cambridge within the program "Highly Oscillatory Problems". The authors acknowledge both Institutions for their hospitality and support. 
$3 \quad L^{2}$-stability and non dissipated solutions $\quad \mathbf{1 5}$

$3.1(\mathrm{SK})$ is rarely satisfied . . . . . . . . . . . . . . . . . 16

3.2 The set of degeneracy . . . . . . . . . . . . . . . . . 16

3.3 A NSC for strong $L^{2}$ stability . . . . . . . . . . . . . . . . . 18

3.4 A NSC for the existence of non dissipated solutions . . . . . . . . 19

3.5 Complete classification in the case $m=1 \ldots \ldots 21$

3.6 The case $\mathcal{D}\left(A_{1}, \ldots, A_{m}, B\right)=\mathbb{R}^{m}$ : traveling waves? . . . . . . . . 22

4 Decomposition of the solutions when $\mathcal{D}$ is the union of vector $\begin{array}{ll}\text { subspaces } & \mathbf{2 4}\end{array}$

4.1 General statement ... . . . . . . . . . . . . . . . 25

4.2 Decomposition when $n_{1}=1 \ldots \ldots$. . . . . . . . . . . 29

4.3 Discussion on explicit examples . . . . . . . . . . . . . . . . . . 31

4.4 Complete classification in the case $n=2 \ldots . . . . . . .34$

5 Summary array of the classification, open problems and conjectures $\mathbf{3} 4$

5.1 Summary array of the classification . . . . . . . . . . . . . 34

5.2 Open problems and conjectures . . . . . . . . . . . 35

6 Global existence around a constant equilibrium for the nonlin$\begin{array}{lr}\text { ear system } & \mathbf{3 6}\end{array}$

6.1 Problem formulation . . . . . . . . . . . . . . . . 36

6.2 Size of the neighborhood for global existence under (SK) . . . . . 37

6.3 Global existence without (SK) . . . . . . . . . . . . . . 41

\section{Introduction and main results}

\subsection{Problem formulation}

This work is concerned with the following $n$-component hyperbolic system of balance laws in $m$ space dimensions:

$$
\frac{\partial w}{\partial t}+\sum_{j=1}^{m} \frac{\partial F_{j}(w)}{\partial x_{j}}=Q(w) .
$$

Here $m, n \in \mathbb{N}^{*}, w: \mathbb{R} \times \mathbb{R}^{m} \rightarrow \mathbb{R}^{n}, w=w(t, x)$ is the unknown and $Q, F_{j}$ : $\mathbb{R}^{n} \rightarrow \mathbb{R}^{n}$ are smooth functions.

Such nonlinear systems typically govern non equilibrium processes in physics, for media with hyperbolic response, as, for example, in gas dynamics. They also arise in the numerical simulation of conservation laws by relaxation schemes (see [1], [3], [8], [24] and references cited therein). In many applications, the source term $Q(w)$ has, or can be transformed by a linear transformation into the form

$$
Q(w)=\left(\begin{array}{c}
0 \\
q(w)
\end{array}\right)
$$

with $0 \in \mathbb{R}^{n_{1}}, q(w) \in \mathbb{R}^{n_{2}}$, where $n_{1}, n_{2} \in \mathbb{N}, n_{1}+n_{2}=n$.

It is well known that (1) has local (in time) smooth solutions (see [15], [19]), but these solutions may develop singularities (i.e. shock waves) in finite time, 
even when the initial data are smooth and small (see [7], [19]). However, in many physical examples, thanks to the interplay between the source term and the flux, there exist global smooth solutions for a suitable set of initial conditions. Total dissipation, which consists in requiring the source damping term to enter in each of the equations of the system distributed all over the space, is a well known assumption for global existence for suitable classes of initial data (see [14]). But this condition is too strong and it is not satisfied by systems (1)-(2) with $n_{1} \neq 0$ (in which the dissipation is not present in all the components of the system) and more general systems with relaxation (see [6], [16]). This is the case for the isentropic Euler system with damping

$$
\frac{\partial u}{\partial t}-\frac{\partial v}{\partial x}=0, \quad \frac{\partial v}{\partial t}+\frac{\partial f(u)}{\partial x}=-v,
$$

with $f^{\prime}(u)<0$ which has been considered in [17] and [13]. In this case, the damping term, even if it enters only in the second equation, may prevent shock formation. We also refer to [18] where similar issues are addressed for systems with linear principal part, which correspond, in particular, to damped wave equations involving nonlinear convective terms.

The existence of global smooth solutions for system (1)-(2) is one of the two main topics of this article. In fact, the study of the nonlinear systems (1) we shall develop relies on a linearization principle, around constant equilibria, and this requires analyzing partially dissipative linear hyperbolic systems of the form

$$
\frac{\partial w}{\partial t}+\sum_{j=1}^{m} A_{j} \frac{\partial w}{\partial x_{j}}=-B w
$$

where $A_{1}, \ldots, A_{m}, B$ are $n * n$ real matrices, $A_{j}:=\left(a_{k, l}^{(j)}\right)_{1 \leqslant k, l \leqslant n}$ being symmetric, for $j=1, \ldots, m$, and $B$ such that

$$
B=\left(\begin{array}{cc}
0 & 0 \\
0 & D
\end{array}\right), \quad D \in \mathbb{R}^{n_{2} \times n_{2}}, \quad X^{t} D X>0, \forall X \in \mathbb{R}^{n_{2}}-\{0\} .
$$

Note that $D$ is not assumed to be symmetric.

The analysis of linear systems of the form (4) is relevant, as mentioned above, to understand the behavior of nonlinear systems and, as we shall see, they may exhibit a very rich behavior. Its analysis is the first goal of this article.

The solutions of (4) with initial conditions $w_{0} \in L^{2}\left(\mathbb{R}^{m}, \mathbb{R}^{n}\right)$ are explicit. Indeed, applying the Fourier transform in the $x$ variable, system (4) can be rewritten as

$$
\frac{\partial \hat{w}}{\partial t}+i \sum_{j=1}^{m} A_{j} \xi_{j} \hat{w}=-B \hat{w}
$$

or

$$
\frac{\partial \hat{w}}{\partial t}(t, \xi)=E(\xi) \hat{w}(t, \xi)
$$

where

$$
E(\xi):=-B-i A(\xi), A(\xi):=\sum_{j=1}^{m} \xi_{j} A_{j} .
$$

Solving this first order ordinary differential equation, we get

$$
\hat{w}(t, \xi)=\exp [E(\xi) t] \hat{w}^{0}(\xi) .
$$


Note that, when $n_{2} \neq n$, the matrix $-E(\xi)$ is not coercive. Indeed, with the notation

$$
X=\left(\begin{array}{c}
X_{1} \\
X_{2}
\end{array}\right) \in \mathbb{C}^{n}, X_{1} \in \mathbb{C}^{n_{1}}, X_{2} \in \mathbb{C}^{n_{2}}, n_{1}+n_{2}=n
$$

we have

$$
X^{t} E(\xi) \bar{X}=-X_{2}^{t} D \bar{X}_{2} .
$$

Thus, this quadratic form does not provide any information on the $X_{1}$ component.

However, it is by now well known, in the context of linear finite-dimensional systems, that this fact is not an obstacle for the solutions of (6) to decay as $t \rightarrow \infty$. Indeed, the interaction of the dissipative operator $B$ with the timedynamics generated by (6) may eventually dissipate all components of solutions. This can be viewed, for instance, through the Kalman rank condition for the control of finite-dimensional systems (see [4] and [25]) that we shall discuss in detail in Section 2.

This fact is also well known in several other contexts, and in particular, for dissipative wave equations [10] which are particular instances of (4), and partially diffusive partial differential equations where the notions of hypoellipticity [11] and hypocoercivity [22] have been introduced to measure the global effect of partial diffusion in the regularity and the time decay of solutions, respectively.

As we shall see, under rather general assumptions on the matrices $A_{1}, \ldots, A_{m}$ and $B$, and, more precisely, under the so called Kalman rank condition for the pair $(A(\xi), B)$, it can be proved that

$$
\exists C>0, \lambda(\xi)>0: \exp [E(\xi) t] \leqslant C e^{-\lambda(\xi) t} .
$$

This decay property, together with explicit estimates on the positivity and dependence of $\lambda(\xi)$ with respect to $\xi$, allows describing accurately the asymptotic behavior of solutions of (4) as $t \rightarrow \infty$, and deriving a decomposition of solutions in which various terms decaying with different rates can be distinguished. Obviously, the overall picture depends in a critical way on the properties of the function $\xi \rightarrow \lambda(\xi)$. The analysis of this function is a complex issue to which we will devote a significant part of this article.

There is in fact an extensive literature on the subject. For instance, in [20], the authors study systems (4) under the so-called Shizuta-Kawashima condition:

$$
\forall \xi \in \mathbb{R}^{m}, \operatorname{Ker}(B) \cap\{\text { eigenvectors of } A(\xi)\}=\{0\},
$$

and prove that

$$
\exists C, c>0 \text { s. t. } \forall \xi \in \mathbb{R}^{m}, \exp [E(\xi) t] \leqslant C e^{-c \min \left\{1,|\xi|^{2}\right\} t} .
$$

Thanks to (9) and (12), the authors deduce that any solution $w$ of (4) associated to an initial condition $w_{0} \in L^{1} \cap L^{2}\left(\mathbb{R}^{m}, \mathbb{R}^{n}\right)$ can be decomposed as

$$
w=w_{1}+w_{2}
$$

where

$$
\begin{aligned}
& \left\|w_{1}(t)\right\|_{L^{2}\left(\mathbb{R}^{m}, \mathbb{R}^{n}\right)} \leqslant C e^{-\lambda t}\left\|w^{0}\right\|_{L^{2}\left(\mathbb{R}^{m}, \mathbb{R}^{n}\right)}, \forall t \in(0,+\infty) \\
& \left\|w_{2}(t)\right\|_{L^{\infty}\left(\mathbb{R}^{m}, \mathbb{R}^{n}\right)} \leqslant C t^{-\frac{m}{2}}\left\|w^{0}\right\|_{L^{1}\left(\mathbb{R}^{m}, \mathbb{R}^{n}\right)}, \forall t \in(0,+\infty)
\end{aligned}
$$


and $C, \lambda$ are positive constants depending only on $A_{1}, \ldots, A_{m}, B$. The two components $w_{1}$ and $w_{2}$ correspond, respectively, to the high and low frequency components. The high frequency component decays exponentially while the low frequency one decays polynomially with the decay rate of the heat kernel. We refer to [18] for similar results for damped wave equations with nonlinear convection.

This result has motivated many others. For instance, in [2], the authors proved more precise decay rates for the high frequency component $w_{1}$ under $(\mathrm{SK})$, and extended the analysis for non linear systems.

Estimate (12) is equivalent to saying that $\lambda(\xi) \geq c \min \left\{1,|\xi|^{2}\right\}$ in (10). In particular, (SK) implies that $\lambda(\xi)$ may only degenerate quadratically at $\xi=0$. Roughly speaking, (SK) is a natural sufficient condition to guarantee that the damping term affects all the components of the system and the $L^{2}$-decay of the solutions of (4) as $t \rightarrow+\infty$. But it is not sharp. Indeed, as we shall see, there are many situations in which $\lambda(\xi)$ degenerates on other points than $\xi=0$, but still the $L^{2}$-decay of solutions holds, together with decompositions in the spirit of (13), but involving extra terms, decaying more slowly than the $m$-dimensional heat kernel. To do that we develop a careful analysis of the finite-dimensional behavior of system (6) in terms of the multi-dimensional parameter $\xi$. This analysis is inspired by control theoretical tools. Indeed, our approach starts from the observation that $(\mathrm{SK})$ holds if the pair of matrices $(A(\xi), B)$ satisfies the Kalman rank condition:

$$
\operatorname{rk}\left[B, A(\xi) B, \ldots, A(\xi)^{n-1} B\right]=n .
$$

The key ingredient to obtain a complete decomposition of solutions is the obtention of a careful measure of the decay rate $\lambda(\xi)$ of solutions of (6) as a function of $\xi$. To do this we construct explicit Lyapunov functionals, taking advantage of the interaction of the matrix $B$ with the dynamics generated by $A(\xi)$. This kind of Lyapunov function is similar to those introduced by $\mathrm{C}$. Villani (see [22]) for the analysis of the decay of partially diffusive systems and is also linked, as we mentioned above, to the extensive literature on damped wave equations (see [10] and [18], for instance).

The approach we develop here, in addition of being more systematic, has also the added advantage of being simpler to be carried out from a technical viewpoint since we avoid some of the long developments in [20] for proving the sufficiency of (SK) to achieve the decomposition above.

Let us now return to the nonlinear systems of balance laws (1). The existence of global smooth solutions in a neighborhood of a constant equilibrium $W_{e} \in \mathbb{R}^{n}$ and $Q\left(W_{e}\right)=0$ was proved in [23], under a suitable dissipation assumption, when the linearized system around $W_{e}$ satisfies (SK).

Let us also mention [9] for the same result in one space dimension $(m=1)$ with many examples of application. We also refer to [5], where the authors consider the multidimensional isothermal Euler equations with a strong relaxation and study the asymptotic behavior of the solutions when the relaxation time tends to zero. They use the arguments of [23] in order to prove the existence of global smooth solutions in a neighborhood of a constant equilibrium, whose size is uniform with respect to the relaxation time.

The techniques we develop in this article, in the frame of [23], allow us to obtain explicit estimates of the size of the neighborhood of $W_{e}$ where the 
existence of global smooth solutions holds. As a consequence of this, using an argument inspired in Coron's return method ([4]), we are able to prove a global existence result around a constant equilibrium that does not fulfill the (SK) condition.

\subsection{Main results}

Let us now describe the content of the article in more detail. Sections 2, 3, 4 and 5 are devoted to the analysis of partially dissipative linear hyperbolic systems and section 6 to the nonlinear systems of balance laws.

More precisely, section 2, is concerned with the Kalman rank condition, the (SK) condition, the notion of hypocoercivity and the decay rates. Subsection 2.1 is devoted to a preliminary discussion in which we show that (SK) is equivalent to the classical Kalman rank condition in control theory for the pairs $(A(\xi), B)$ and all $\xi \neq 0$. Then we develop the proof of decay (10) using Lyapunov functionals (see subsection 2.2). In particular, this yields a more systematic approach and a simpler way to get the decomposition (13)-(14) in [20] under the condition (SK) (see subsection 2.3).

In section 3, using the tools developed in section 2, we investigate the asymptotic behavior for (4) without the (SK) condition. More precisely, we study the $L^{2}$-stability and the non dissipated solutions of (4). For doing that we introduce the set of degeneracy

$$
\mathcal{D}\left(A_{1}, \ldots, A_{m}, B\right):=\left\{\xi \in \mathbb{R}^{m}: \operatorname{Ker}(B) \cap\{\text { eigenvectors of } A(\xi)\} \neq\{0\}\right\},
$$

i. e. the set of values of $\xi$ for which (SK) fails.

Under the (SK) condition this set is the trivial one $\{0\}$. But, of course, this is false in general and, as we shall see, the structure of this set determines the asymptotic behavior of solutions. In subsection 3.2, using the fact that the Kalman condition fails on the set of degeneracy when (SK) does not hold, we prove that, either

- $\mathcal{D}\left(A_{1}, \ldots, A_{m}, B\right)$ is a strict algebraic submanifold of $\mathbb{R}^{m}$,

or

- $\mathcal{D}\left(A_{1}, \ldots, A_{m}, B\right)=\mathbb{R}^{m}$.

In subsection 3.3, we prove that, in the first case, (4) is strongly stable in $L^{2}$, i. e. all $L^{2}$ solutions tend to zero in $L^{2}$ as $t \rightarrow \infty$. In subsection 3.4 , we prove that, in the second case, there exist non dissipated solutions of constant $L^{2}$-norm.

In subsection 3.5 , we study the $1 \mathrm{D}$ case $m=1$ whatever the size $n$ of the system is. We show that, in this case, the (SK) condition is sharp in the sense that it characterizes completely the behavior of the solutions :

- $(\mathrm{SK})$ is a necessary and sufficient condition for the strong stability in $L^{2}\left(\mathbb{R}, \mathbb{R}^{n}\right)$,

- (SK) is a necessary and sufficient condition for the decomposition (14), 
- without (SK), any solution associated to an $L^{2}$ initial condition can be decomposed as $w=w_{1}+w_{2}+w_{t r w}$ where $w_{1}, w_{2}$ satisfy (14) and $w_{t r w}$ is the sum of a finite number of traveling waves.

This shows, in particular, that in $1 \mathrm{D}$ we may not have a third component decaying at infinity with a slower decay rate. The latter may only arise in the multi-dimensional case.

Subsection 3.6 is devoted to analyze the most degenerate case in which $\mathcal{D}\left(A_{1}, \ldots, A_{m}, B\right)=\mathbb{R}^{m}$. We show that this is a necessary condition for the existence of traveling waves with $L^{2}$ profiles. However, contrary to the case $n=1$, this condition fails to be sufficient when $m \geqslant 2$. Indeed, the solutions of constant energy may, in general, have a more complex structure that being sums of traveling waves.

In section 4, we investigate the analogue of the decomposition (13)-(14) for the solutions of (4), when (SK) is not fulfilled and when the measure of the set of degeneracy $\mathcal{D}\left(A_{1}, \ldots, A_{m}, B\right)$ vanishes. We answer this question in some particular cases where the set of degeneracy is the union of a finite number of vector subspaces.

In subsection 4.1, we prove a general statement giving a decomposition for the solutions of (4) when the set of degeneracy $\mathcal{D}\left(A_{1}, \ldots, A_{m}, B\right)$ is the union of a finite number of vector subspaces and under an assumption of the following type

$$
N_{*}(\omega) \geqslant C \operatorname{dist}\left(\omega, \mathcal{D}\left(A_{1}, \ldots, A_{m}, B\right)\right)^{\alpha}, \forall \omega \in S^{m-1},
$$

for some constants $C>0$ and $\alpha \geqslant 2$, where

$$
N_{*}(\omega):=\min \left\{\sum_{k=0}^{n-1} \epsilon^{m_{k}}\left|B A(\omega)^{k} x\right|^{2} ; x \in S^{n-1}\right\},
$$

for a suitable small enough $\epsilon$ and suitable exponents $m_{k}$ (see Proposition 1). Note that the value of $N_{*}(\omega)$ provides a quantitative version of the (SK) property, or the Kalman rank property, in the sense that, when it fails on an isolated point $\omega$, it holds in the neighboring ones (that do not belong to $\mathcal{D}\left(A_{1}, \ldots, A_{m}, B\right)$ ), with an explicit lower bound on $N_{*}$ as a power of the distance function.

This decomposition is of the form $w=w_{1}+w_{2}+w_{3}+w_{4}$ where $w_{1}$ and $w_{2}$ satisfy (14) and $w_{3}$ (resp. $w_{4}$ ) contains some (but not all) high (resp. low) frequencies and decays like $t^{-1 / \alpha}$ (resp. $t^{-r / \alpha}$ where $r \in \mathbb{N}^{*}$ ).

In this way we see that, in dimension $m \geq 2$, there is a whole class of phenomena that do not arise under the condition (SK). Note that we already got a complete classification of the possible decompositions for the solutions of (4) when $m=1$ in subsection 3.5 .

In subsection 4.2 , we study the particular case $n_{1}=1$ (i.e. $\operatorname{Ker}(B)=$ $\left.\operatorname{Span}\left(e_{1}\right)\right)$ in which the condition on the set of degeneracy being the union of vector subspaces is automatically satisfied. In that case, (16) holds with a smaller exponent $\alpha=2$. This leads to a decomposition for the solutions of (4), in this particular case $n_{1}=1$.

In subsection 4.3, considering explicit examples, we show that there are situations in which $\alpha=4$ is the smallest exponent one can have in (16), and the parameter $r$ may take any positive value. 
In subsection 4.4, we deduce from the previous analysis a complete classification in the case $n=2$, whatever the space dimension $m$ is.

In section 5, we recapitulate the various results obtained in the previous sections, presenting them in a table. This classification is still incomplete in the sense that not all possible values of $m$ and $n$ are covered by our analysis. Indeed, we do not provide a decomposition

- in the general case where the set of degeneracy is the union of a finite number of vector subspaces because we only study particular examples,

- when the set of degeneracy $\mathcal{D}\left(A_{1}, \ldots, A_{m}, B\right)$ is an algebraic submanifold that is not a union of vector subspaces,

- or when $\mathcal{D}\left(A_{1}, \ldots, A_{m}, B\right)$ is the whole space.

We also make precise some open questions and some conjectures about the cases that our partial classification does not cover.

In Section 6, we study the existence of global (in time) smooth solutions for the non linear system (1), locally around a constant equilibrium $W_{e} \in \mathbb{R}^{n}$. In subsection 6.1 we make precise the context of our work. The novelty of our study with respect to [23] is that we do not impose the (SK) condition on the linearized system around $W_{e}$. To do that we proceed in two steps. We first assume that (SK) holds and taking advantage of the explicit Lyapunov function introduced in subsection 2.2, we make the result in [23] more precise by giving an explicit estimate on the size for the neighborhood of $W_{e}$ on which global existence holds. Then, in subsection 6.3, using these explicit estimates and under some suitable assumptions on the nonlinearities, we prove the existence of global solutions for (1), around a constant equilibrium $W_{e}$ that does not satisfy (SK). This is done assuming the existence of a family of constant equilibria fulfilling (SK) converging to $W_{e}$. This approach is inspired by Coron's return method for the controllability of nonlinear systems (see [4] for an introduction and examples of applications), that takes advantage of the nonlinearity of the system. We conclude subsection 6.3 with an example of application of the previous theorem.

At this point, it is convenient to note that the fact that (SK) was not necessary for the existence of global smooth solutions around a constant equilibrium was already known. Indeed, Zeng proved in [24] the existence of global 1D $(m=1)$ smooth solutions for an equation of gas dynamics, around a constant state, without the (SK) condition, splitting the system in two parts, one of them being linearly degenerate. But, to our knowledge, there were no general result of global existence for partially dissipative hyperbolic systems without (SK) (for arbitrary $m, n)$. Our result is the first one in that direction. One may expect that a further analysis of the linearized systems along the lines we do in sections $2,3,4$ together with this way of approaching the nonlinear one, will allow to generalize further the result we are presenting here.

Notations: In this article, $\Re(),. \Im($.$) denote the real and imaginary parts of$ complex numbers, $\left(e_{1}, \ldots, e_{n}\right)$ is the canonical basis of $\mathbb{R}^{n}$ and $\langle.,$.$\rangle denotes the$ hermitian product in $\mathbb{C}^{n},\langle X, Y\rangle=X^{t} \bar{Y}$.

The notations given in this introduction $\left(m, n, n_{1}, n_{2}, A_{j}=\left(a_{k, l}^{(j)}\right)_{1 \leqslant k, l \leqslant n}\right.$, $\left.B, D, \mathcal{D}\left(A_{1}, \ldots, A_{m}, B\right)\right)$ will be used all along the paper. 


\section{Rank conditions, (SK), hypocoercivity and de- cay rates}

In subsection 2.1, we show the equivalence between the (SK) condition for hyperbolic systems and the Kalman rank condition in control theory. In subsection 2.2 , taking advantage of this equivalence, we prove an explicit decay rate for $\lambda(\xi)$ in (10), by means of a Lyapunov functional. Finally, in subsection 2.3, we recover Shizuta-Kawashima's decomposition (13)-(14) for the solutions of (4) under the (SK) condition.

\section{1 (SK) and Kalman rank conditions}

The following lemma is one of the key ingredients of this section.

Lemma 1 Let $n \in \mathbb{N}^{*}, A, B$ be $n * n$ matrices with real coefficients, such that $B$ has the form (5). The following statements are equivalent

- (1) $A$ and $B$ satisfy $(S K):\{$ eigenvectors of $A\} \cap \operatorname{Ker}(B)=\{0\}$,

- (2) $\forall y \in \mathbb{C}^{n}-\{0\}, t \mapsto B \exp (A t) y$ does not vanish on $\mathbb{R}$,

- (3) $\forall y \in \mathbb{C}^{n}-\{0\}$, there exists $k \in\{0,1, \ldots, n-1\}$ such that $B A^{k} y \neq 0$,

- (4) for every $a_{0}, \ldots, a_{n-1}>0$, the expression

$$
N(y):=\left(\sum_{k=0}^{n-1} a_{k}\left|B A^{k} y\right|^{2}\right)^{1 / 2}
$$

defines a norm on $\mathbb{C}^{n}$

- (5) $(A, B)$ satisfies the Kalman rank condition : the $\left(n^{2}\right) * n$ Kalman matrix

$$
K:=\left(\begin{array}{c}
B \\
B A \\
\cdots \\
B A^{n-1}
\end{array}\right)
$$

has $\operatorname{rank} n$.

These equivalences are classical (see for example [4, Chapter 1.3], [21, Theorem 2.2.1]). We give a proof for the sake of completeness.

Proof of Lemma 1: Let us prove "(1) $\Rightarrow(\mathbf{2})$ ". We assume that (2) is false. Then, there exists $y \in \mathbb{C}^{n}-\{0\}$ such that

$$
B \exp (A t) y \equiv 0 .
$$

Let

$$
\operatorname{det}\left(A-X I_{n}\right):=\prod_{j=1}^{\nu}\left(X-\lambda_{j}\right)^{r_{j}}
$$


be the characteristic polynomial of $A$. We have

$$
\begin{aligned}
\mathbb{C}^{n} & =\bigoplus_{j=1}^{\nu} \operatorname{Ker}\left[\left(A-\lambda_{j}\right)^{r_{j}}\right] \\
y & =\sum_{j=1}^{\nu} y_{j} .
\end{aligned}
$$

The equality (19) gives

$$
\sum_{j=1}^{\nu} e^{\lambda_{j} t} \sum_{k=0}^{r_{j}-1} \frac{t^{k}}{k !} B\left(A-\lambda_{j}\right)^{k} y_{j} \equiv 0 .
$$

Thanks to the linear independence of the family

$$
\left\{t^{k} e^{\lambda_{j} t} ; 0 \leqslant k \leqslant r_{j}-1,1 \leqslant j \leqslant \nu\right\}
$$

we deduce that

$$
B\left(A-\lambda_{j}\right)^{k} y_{j}=0, \forall k \in\left\{0, \ldots, r_{j}-1\right\}, \forall j \in\{1, \ldots, \nu\} .
$$

Let $j \in\{1, \ldots, \nu\}$ be such that $y_{j} \neq 0$ and $k \in\left\{0, \ldots, r_{j}-1\right\}$ be the largest integer such that $\left(A-\lambda_{j}\right)^{k} y_{j} \neq 0$. Then, $\left(A-\lambda_{j}\right)^{k} y_{j}$ is an eigenvector of $A$ in the null space of $B$. Thus, (1) is false.

Let us prove now that " $(\mathbf{2}) \Rightarrow(\mathbf{3})$ ". We assume that (3) is false. Then, there exists $y \in \mathbb{C}^{n}-\{0\}$ such that $B A^{k} y=0$ for $k=0, \ldots, n-1$ and thanks to the Cayley-Hamilton theorem, $B A^{k} y=0$ for every $k \in \mathbb{N}$. Thus $B \exp (A t) y \equiv 0$ and (2) is false.

The implication " $(3) \Rightarrow(4)$ " is clear.

Let us prove "(4) $\Rightarrow(5)$ ". We assume (4). If $y \in \operatorname{Ker}(K)$, then $B A^{k} y=0$ for $k=0, \ldots, n-1$. Thus $N(y)=0$ and so $y=0$. This proves that $K$ is injective and thus $\operatorname{rk}(K)=n$.

Finally, let us prove "(5) $\Rightarrow(\mathbf{1})$ ". We assume (1) is false. Let $v \in S^{n-1}$ be an eigenvector of $A$ in the null space of $B$. Then $K y=0$ and, thus, $K$ is not injective. Therefore $\operatorname{rk}(K)<n$, i.e. (5) is false.

\subsection{Lyapunov functionals and explicit decay rates}

According to the results of the previous section there is a very explicit connection between the (SK) condition, which arises naturally in the analysis of the decay of partially dissipative hyperbolic systems, and the Kalman rank condition in finite dimensional control theory.

In this subsection we develop one of the key elements of this article which consists in deriving explicit decay rates for the finite-dimensional system (6) in terms of $\xi$. To do this we construct explicit Lyapunov functionals, which are inspired on those rank conditions and more precisely in the statement (4) of Lemma 1.

We set $\xi=\rho \omega$, with $\rho>0$ and $\omega \in S^{m-1}$, and, consequently, rewrite (6) in the form:

$$
\dot{x}=-(B+i \rho A(\omega)) x, \quad x(0)=x_{0} \in \mathbb{C}^{n} .
$$


Proposition 1 We fix a family of non-negative real numbers $\left(m_{k}\right)_{0 \leqslant k \leqslant n}$ such that

$$
\begin{gathered}
0=m_{0}<m_{1}<\ldots<m_{n}, \\
m_{k}-\frac{m_{k-1}+m_{k+1}}{2} \geqslant \delta>0, \forall k=1, \ldots, n-1,
\end{gathered}
$$

for some $\delta>0$.

Let $A_{1}, \ldots, A_{m}, B$ be $n * n$ real matrices such that $B$ has form (5). For $\omega \in S^{m-1}, \epsilon>0$ we define the symmetric non-negative matrix

$$
M_{\epsilon}(\omega):=\sum_{k=0}^{n-1} \epsilon^{m_{k}}\left(A(\omega)^{t}\right)^{k} B^{t} B A(\omega)^{k}
$$

and its minimal eigenvalue

$$
N_{*, \epsilon}(\omega):=\min \left\{\left\langle x, M_{\epsilon}(\omega) x\right\rangle ; x \in S^{n-1}\right\}=\min \left\{\sum_{k=0}^{n-1} \epsilon^{m_{k}}\left|B A(\omega)^{k} x\right|^{2} ; x \in S^{n-1}\right\} .
$$

Then, there exist $\epsilon_{*}=\epsilon_{*}\left(A_{1}, \ldots, A_{m}, B\right) \in(0,1)$ and $\tilde{c}=\tilde{c}\left(A_{1}, \ldots, A_{m}, B\right)>0$ such that, for every $\epsilon \in\left(0, \epsilon_{*}\right), x_{0} \in \mathbb{C}^{n}, \rho \in(0,+\infty)$ and $\omega \in S^{m-1}$, the solution of (20) satisfies

$$
|x(t)| \leqslant \sqrt{3}\left|x_{0}\right| e^{-\tilde{c} N_{*, \epsilon}(\omega) \min \left\{1, \rho^{2}\right\} t}, \forall t \in(0,+\infty) .
$$

Remark 1 This result provides an exponential decay rate for $\exp [E(\rho \omega) t]$, which is explicit in terms of $\rho$ and $\omega$, and more precisely on $N_{*, \epsilon}(\omega)$. As we will see in sections 2.3 and 4, the main interest of this Proposition is that it reduces the problem of the asymptotic behavior of the solutions of (4) to the study of the real valued map $\omega \in S^{m-1} \mapsto N_{*, \epsilon}(\omega) \in \mathbb{R}_{+}$.

Note that Proposition 1 holds without assuming the (SK) condition, which, in fact, only enters when trying to obtain a uniform lower bound on $N_{*, \epsilon}(\omega)$ for $\omega \in S^{m-1}$, in section 2.3. In particular, it could be that, for some value $\omega^{*}$ of $\omega, N_{*, \epsilon}\left(\omega^{*}\right)=0$. In that case, to get explicit decay rates for (4), one has to analyze the behavior of $N_{*, \epsilon}(\omega)$ for values $\omega$ close to $\omega^{*}$. This fact will play an important role when deriving decompositions of solutions in the absence of the (SK) condition and analyzing their decay rates as $t \rightarrow \infty$ in section 4.

Remark 2 The constant $\sqrt{3}$ in (25) can be replaced by any constant $\tilde{C}>1$. Indeed, an easy adaptation of the proof below provides the following more general statement: for every $\tilde{C}>1$, there exist $\epsilon_{*}=\epsilon_{*}\left(A_{1}, \ldots, A_{m}, B\right)>0$ and $\tilde{c}=$ $\tilde{c}\left(\tilde{C}, A_{1}, \ldots, A_{m}, B\right)>0$ such that the same conclusion holds with $\sqrt{3}$ replaced by $\tilde{C}$.

On the other hand, $\tilde{C}$ being fixed, it is natural to raise the issue of finding the value of $\epsilon$ that maximizes the decay rate. This is an open problem.

Remark 3 Notice that Proposition 1 holds without assuming the symmetry of the matrices $A_{1}, \ldots, A_{m}$. This will be used in section 6 .

Proof of Proposition 1: First, let us introduce some notations. Since $B$ has the form (5), there exists $C_{1}=C_{1}(B)>0$ such that

$$
\Re\langle B x, x\rangle \geqslant C_{1}|B x|^{2}, \forall x \in \mathbb{C}^{n} .
$$


We consider the characteristic polynomial

$$
\operatorname{det}\left(X I_{n}-A(\omega)\right)=X^{n}+\sum_{k=0}^{n-1} a_{k}(\omega) X^{k},
$$

$a_{k}(\omega)$ being the coefficient of its $k-t h$ order term. We also set

$$
\begin{gathered}
\mathcal{M}_{1}:=\max \left\{\left\|B A(\omega)^{k}\right\|: 0 \leqslant k \leqslant n-1, \omega \in S^{m-1}\right\}, \\
\mathcal{M}_{2}:=\max \left\{\left|a_{k}(\omega)\right|: 0 \leqslant k \leqslant n-1, \omega \in S^{m-1}\right\} .
\end{gathered}
$$

Let $\epsilon_{*}=\epsilon_{*}\left(A_{1}, \ldots, A_{m}, B\right)>0$ be small enough so that, for every $\omega \in S^{m-1}$,

$$
\begin{gathered}
\sum_{k=1}^{n-1} \epsilon_{*}^{m_{k}}\left\|\left(A(\omega)^{t}\right)^{k} B^{t} B A(\omega)^{k-1}\right\|<\frac{1}{2}, \\
\mathcal{M}_{1} \epsilon_{*}^{m_{1}}+\epsilon_{*}^{\delta}+n \mathcal{M}_{2}^{2} \epsilon_{*}^{\delta}<\frac{C_{1}}{2}, \\
\frac{\mathcal{M}_{1}^{2}(n-1)}{C_{1}} \epsilon_{*}^{m_{1}}+\epsilon_{*}^{\delta}\left(1+n \mathcal{M}_{2}^{2}\right)<\frac{1}{2} .
\end{gathered}
$$

For $\epsilon \in\left(0, \epsilon_{*}\right), \rho \in(0,+\infty), \omega \in S^{m-1}$ and $x \in \mathbb{C}^{n}$ we consider

$$
\mathcal{L}_{\rho, \omega, \epsilon}(x):=|x|^{2}+\min \left\{\rho, \frac{1}{\rho}\right\} \sum_{k=1}^{n-1} \epsilon^{m_{k}} \Im\left\langle B A(\omega)^{k-1} x, B A(\omega)^{k} x\right\rangle .
$$

Thanks to (29), one has

$$
\frac{1}{2}|x|^{2} \leqslant \mathcal{L}_{\rho, \omega, \epsilon}(x) \leqslant \frac{3}{2}|x|^{2}, \forall x \in \mathbb{C}^{n}, \forall \rho \in(0,+\infty), \forall \omega \in S^{m-1}, \forall \epsilon \in\left(0, \epsilon_{*}\right) .
$$

Let $\epsilon \in\left(0, \epsilon_{*}\right)$. In order to simplify the notations and since $\epsilon$ is fixed, in the sequel, we will rather write $N_{*}(\omega)$ and $\mathcal{L}_{\rho, \omega}$ instead of $N_{*, \epsilon}(\omega)$ and $\mathcal{L}_{\rho, \omega, \epsilon}$.

When $x$ solves $(20)$, one has

$$
\begin{aligned}
\frac{d}{d t}\left[\mathcal{L}_{\rho, \omega}(x)\right]= & -2 \Re\left(\left\langle\left(B+i \rho A_{\omega}\right) x, x\right\rangle\right) \\
& -\min \left\{\rho, \frac{1}{\rho}\right\} \sum_{k=1}^{n-1} \epsilon^{m_{k}} \Im\left(\left\langle B A_{\omega}^{k-1}\left(B+i \rho A_{\omega}\right) x, B A_{\omega}^{k} x\right\rangle\right) \\
& \left.-\min \left\{\rho, \frac{1}{\rho}\right\} \sum_{k=1}^{n-1} \epsilon^{m_{k}} \Im\left(B A_{\omega}^{k-1} x, B A_{\omega}^{k}\left(B+i \rho A_{\omega}\right) x\right\rangle\right)
\end{aligned}
$$

where $A_{\omega}:=A(\omega)$.

We now distinguish two cases: $0<\rho<1$ and $\rho>1$.

The case $\rho \in(0,1)$ : Using $(26)$, we get

$$
\begin{aligned}
\frac{d}{d t}\left[\mathcal{L}_{\rho, \omega}(x)\right] \leqslant & -2 C_{1}|B x|^{2}-\rho^{2} \sum_{k=1}^{n-1} \epsilon^{m_{k}}\left|B A_{\omega}^{k} x\right|^{2} \\
& +\rho \sum_{k=1}^{n-1} \epsilon^{m_{k}}|B x|\left[\left\|B A_{\omega}^{k-1}\right\|\left|B A_{\omega}^{k} x\right|+\left\|B A_{\omega}^{k}\right\|\left|B A_{\omega}^{k-1} x\right|\right] \\
& +\rho^{2} \sum_{k=1}^{n-1} \epsilon^{m_{k}}\left|B A_{\omega}^{k-1} x \| B A_{\omega}^{k+1} x\right| .
\end{aligned}
$$

Moreover, by (27) and (21) we get

$$
\begin{aligned}
& \rho \sum_{k=1}^{n-1} \epsilon^{m_{k}}|B x|\left[\left\|B A_{\omega}^{k-1}\right\|\left|B A_{\omega}^{k} x\right|+\left\|B A_{\omega}^{k}\right\|\left|B A_{\omega}^{k-1} x\right|\right] \\
\leqslant & \mathcal{M}_{1} \rho \sum_{k=1}^{n-1} \epsilon^{m_{k}}|B x|\left[\left|B A_{\omega}^{k} x\right|+\left|B A_{\omega}^{k-1} x\right|\right] \\
\leqslant & \mathcal{M}_{1} \rho \epsilon^{m_{1}}|B x|^{2}+\sum_{k=1}^{n-1} 2 \mathcal{M}_{1} \rho \epsilon^{m_{k}}|B x|\left|B A_{\omega}^{k} x\right| \\
\leqslant & \mathcal{M}_{1} \rho \epsilon^{m_{1}}|B x|^{2}+\sum_{k=1}^{n-1}\left(\frac{C_{1}}{n-1}|B x|^{2}+\rho^{2} \frac{\mathcal{M}_{1}^{2}(n-1)}{C_{1}} \epsilon^{2 m_{k}}\left|B A_{\omega}^{k} x\right|^{2}\right) \\
\leqslant & \left(C_{1}+\mathcal{M}_{1} \rho \epsilon^{m_{1}}\right)|B x|^{2}+\rho^{2} \frac{\mathcal{M}_{1}^{2}(n-1)}{C_{1}} \sum_{k=1}^{n-1} \epsilon^{2 m_{k}}\left|B A_{\omega}^{k} x\right|^{2} .
\end{aligned}
$$


Using (22), we get

$$
\begin{aligned}
& \rho^{2} \sum_{k=1}^{n-1} \epsilon^{m_{k}}\left|B A_{\omega}^{k-1} x\right|\left|B A_{\omega}^{k+1} x\right| \\
\leqslant & \rho^{2} \epsilon^{\delta} \sum_{k=1}^{n-1} \epsilon^{\frac{m_{k-1}+m_{k+1}}{2}}\left|B A_{\omega}^{k-1} x\right|\left|B A_{\omega}^{k+1} x\right| \\
\leqslant & \frac{1}{2} \rho^{2} \epsilon^{\delta} \sum_{k=1}^{n-1} \epsilon^{m_{k-1}}\left|B A_{\omega}^{k-1} x\right|^{2}+\epsilon^{m_{k+1}}\left|B A_{\omega}^{k+1} x\right|^{2} \\
\leqslant & \rho^{2} \epsilon^{\delta} \sum_{k=0}^{n} \epsilon^{m_{k}}\left|B A_{\omega}^{k} x\right|^{2} .
\end{aligned}
$$

Thanks to Cayley-Hamilton theorem, Cauchy-Schwartz inequality, (28) and (21), the last term of the right hand side in the previous inequality satisfies

$$
\begin{aligned}
\rho^{2} \epsilon^{\delta+m_{n}}\left|B A_{\omega}^{n} x\right|^{2} & =\rho^{2} \epsilon^{\delta+m_{n}}\left|\sum_{k=0}^{n-1} a_{k}(\omega) B A_{\omega}^{k} x\right|^{2} \\
& \leqslant n \mathcal{M}_{2}^{2} \rho^{2} \epsilon^{\delta+m_{n}} \sum_{k=0}^{n-1}\left|B A_{\omega}^{k} x\right|^{2} \\
& \leqslant n \mathcal{M}_{2}^{2} \rho^{2} \epsilon^{\delta} \sum_{k=0}^{n-1} \epsilon^{m_{k}}\left|B A_{\omega}^{k} x\right|^{2}
\end{aligned}
$$

Finally, using (36), (37) and (38) in (35) and thanks to (30), (31), we get

$$
\frac{d}{d t}\left[\mathcal{L}_{\rho, \omega}(x)\right] \leqslant-\frac{C_{1}}{2}|B x|^{2}-\frac{\rho^{2}}{2} \sum_{k=1}^{n-1} \epsilon^{m_{k}}\left|B A_{\omega}^{k} x\right|^{2} .
$$

Therefore, using (24) and (33) we get

$$
\frac{d}{d t}\left[\mathcal{L}_{\rho, \omega}(x(t))\right] \leqslant-2 \tilde{c} N_{*}(\omega) \rho^{2} \mathcal{L}_{\rho, \omega}(x(t))
$$

where

$$
\tilde{c}:=\frac{1}{6} \min \left\{C_{1}, 1\right\} .
$$

Finally, thanks to (33), we get

$$
|x(t)| \leqslant \sqrt{3}\left|x_{0}\right| e^{-\tilde{c} N_{*}(\omega) \rho^{2} t} .
$$

The case $\rho \in(1,+\infty)$ : Now, let us justify the decay estimate in Proposition 1 for $\rho \in(1,+\infty)$. Let $\rho \in(1,+\infty)$ and $\omega \in S^{m-1}$. When $x$ solves (20), with the same arguments as in the previous case, we get

$$
\begin{aligned}
\frac{d}{d t}\left[\mathcal{L}_{\rho, \omega}(x(t))\right] \leqslant & -2 C_{1}|B x|^{2}-\sum_{k=1}^{n-1} \epsilon^{m_{k}}\left|B A_{\omega}^{k} x\right|^{2} \\
& +\frac{1}{\rho} \sum_{k=1}^{n-1} \epsilon^{m_{k}}|B x|\left[\left\|B A_{\omega}^{k-1}\right\|\left|B A_{\omega}^{k} x\right|+\left\|B A_{\omega}^{k}\right\|\left|B A_{\omega}^{k-1} x\right|\right] \\
& +\sum_{k=1}^{n-1} \epsilon^{m_{k}}\left|B A_{\omega}^{k-1} x \| B A_{\omega}^{k+1} x\right|
\end{aligned}
$$

and

$$
\frac{d}{d t}\left[\mathcal{L}_{\rho, \omega}(x(t))\right] \leqslant-\frac{C_{1}}{2}|B x|^{2}-\frac{1}{2} \sum_{k=1}^{n-1} \epsilon^{m_{k}}\left|B A_{\omega}^{k} x\right|^{2} .
$$

Note that the estimates we get are very close to those of the case $\rho \in(0,1)$ except for the fact that, due to the weight $1 / \rho$ on the second term in (32), all terms in which $\rho$ appears have to be divided by $\rho$.

Finally, with the same computations as in the first case, we get

$$
|x(t)| \leqslant \sqrt{3}\left|x_{0}\right| e^{-\tilde{c} N_{*}(\omega) t}
$$


Remark 4 The explicit Lyapunov function (32) is inspired by those introduced by Villani in [22] to derive decay estimates for partially diffusive systems. In [22] the operators under consideration are of the form $\mathcal{L}=\mathcal{A}^{*} \mathcal{A}+\mathcal{B}$ where $\mathcal{B}$ is antisymmetric. In our case the operator has rather the form $L=B+i \rho A(\omega)$ where $i \rho A(\omega)$ is antisymmetric but $B$ does not necessarily have any symmetry property.

The Lyapunov functional we use is also similar to those used in the analysis of the decay properties of dissipative wave equations, as, for instance, $v_{t t}-\Delta v+$ $v_{t}=0$. There the systems under consideration are second order (in time), and in the Fourier setting they take the form of the following dissipated harmonic oscillator $\hat{x}^{\prime \prime}+|\xi|^{2} \hat{x}+\hat{x}^{\prime}=0$. The energy of the system is then given by

$$
e(t)=\frac{1}{2}\left[|\hat{x}|^{2}+\left|\hat{x}^{\prime}\right|^{2}\right]
$$

while the Lyapunov functional to be used to derive the decay is of the form

$$
\mathcal{L}(t)=\frac{1}{2}\left[|\hat{x}|^{2}+\left|\hat{x}^{\prime}\right|^{2}\right]+\varepsilon \hat{x} \hat{x}^{\prime} .
$$

This corresponds precisely to functionals of the form (32) in the particular case in which $n=2$.

\subsection{A new proof for Shizuta and Kawashima's decompo- sition}

As a consequence of Proposition 1, the decomposition (14) is straightforward.

Theorem 1 We assume that $B$ has form (5), $A_{1}, \ldots, A_{m}$ are symmetric and (SK) is satisfied. Then, there exist

$$
C=C\left(A_{1}, \ldots, A_{m}, B\right), \lambda=\lambda\left(A_{1}, \ldots, A_{m}, B\right)>0
$$

such that, for every $w^{0} \in L^{1} \cap L^{2}\left(\mathbb{R}^{m}, \mathbb{R}^{n}\right)$, the solution $w(t, x)$ of (4) can be decomposed as in (13) where (14) holds.

Proof of Theorem 1 : Let $\epsilon_{*}>0$ be as in Proposition 1 and $\epsilon \in\left(0, \epsilon_{*}\right)$. Thanks to the implication "(1) $\Rightarrow(\mathbf{4})$ " in Lemma 1 , and in view of the (SK) assumption, we have $N_{*, \epsilon}(\omega)>0$ for every $\omega \in S^{m-1}$. Moreover, the function $\omega \mapsto N_{*, \epsilon}(\omega)$ is continuous on the compact set $S^{m-1}$ and, therefore, there exists $N_{*}>0$ such that $N_{*, \epsilon}(\omega) \geqslant N_{*}$, for every $\omega \in S^{m-1}$. We define $w_{1}$ and $w_{2}$ by

$$
\hat{w}_{1}(t, \xi):=\hat{w}_{1}(t, \xi) 1_{|\xi|>1} \quad \text { and } \quad \hat{w}_{2}(t, \xi):=\hat{w}_{1}(t, \xi) 1_{|\xi|<1} .
$$

Tanks to Proposition 20, one has,

$$
\left|\hat{w}_{1}(t, \xi)\right| \leqslant \sqrt{3}\left|\hat{w}^{0}(\xi)\right| e^{-\tilde{c} N_{*} t}, \quad\left|\hat{w}_{2}(t, \xi)\right| \leqslant \sqrt{3}\left|\hat{w}^{0}(\xi)\right| e^{-\tilde{c} N_{*}|\xi|^{2} t},
$$

for all $\xi \in \mathbb{R}^{m}$ and $t \in(0,+\infty)$. Thus, one has

$$
\begin{aligned}
\left\|w_{1}(t)\right\|_{L^{2}\left(\mathbb{R}^{m}, \mathbb{R}^{n}\right)} & \leqslant \sqrt{3}\left\|w^{0}\right\|_{L^{2}\left(\mathbb{R}^{m}, \mathbb{R}^{n}\right)} e^{-\tilde{c} N_{*} t}, \\
\left\|w_{2}(t)\right\|_{L^{\infty}\left(\mathbb{R}^{m}, \mathbb{R}^{n}\right)} & \leqslant\left\|\hat{w}_{2}(t)\right\|_{L^{1}\left(\mathbb{R}^{m}, \mathbb{R}^{n}\right)} \\
& \leqslant \sqrt{3} \int_{\mathbb{R}^{m}}\left|\hat{w}^{0}(\xi)\right| e^{-\tilde{c} N_{*}|\xi|^{2} t} d \xi \\
& \leqslant C t^{-m / 2}\left\|w^{0}\right\|_{L^{1}\left(\mathbb{R}^{m}, \mathbb{R}^{n}\right)} .
\end{aligned}
$$


Remark 5 Let us compare the tools developed here with those in [20]. In [20], the authors use algebraic tools to justify the equivalence between (SK) and the existence of a compensating function (notion defined below) for (4). Then, they use this compensating function to prove the decomposition of Proposition 1 with an energy approach.

Definition $1 A C^{\infty}$ map $\omega \in S^{m-1} \mapsto K(\omega) \in \mathbb{C}^{n * n}$ is a compensating function for (4) if

- $K(-\omega)=-K(\omega), \forall \omega \in S^{m-1}$,

- $K(\omega)$ is a skew-symmetric matrix, for every $\omega \in S^{m-1}$,

- $\frac{B^{*}+B}{2}+\frac{1}{2}\left(K(\omega) A(\omega)+(K(\omega) A(\omega))^{t}\right)$ is positive definite for every $\omega \in$

The proof of Proposition 1 contains the arguments to justify that, when $\epsilon^{\delta}(1+$ $\left.n \mathcal{M}_{2}^{2}\right)<1 / 2$, the expression

$$
K(\omega):=\sum_{k=1}^{n-1} \epsilon^{m_{k}}\left[\left(A(\omega)^{t}\right)^{k} B^{t} B A(\omega)^{k-1}-\left(A(\omega)^{t}\right)^{k-1} B^{t} B A(\omega)^{k}\right]
$$

defines a compensating function and that

$$
\frac{B^{*}+B}{2}+\frac{1}{2}\left(K(\omega) A(\omega)+(K(\omega) A(\omega))^{*}\right) \geqslant \frac{1}{2} \min \left\{C_{1}, 1\right\} N_{*, \epsilon}(\omega) .
$$

Notice that, once a compensating function $K(\omega)$ is known, the expression

$$
\tilde{\mathcal{L}}_{\epsilon}(x):=|x|^{2}+\epsilon \min \{1, \rho\} \Im\langle K(\omega) x, x\rangle
$$

for $\epsilon>0$ small enough, provides a Lyapunov function for the proof of Proposition 1 .

Our proof is however much more direct and yields the desired decay rate by means of an explicit Lyapunov function, inspired by Lemma 1.

\section{$3 \quad L^{2}$-stability and non dissipated solutions}

This section is devoted to the study of the $L^{2}$-stability and the non dissipated solutions of (4) when $(\mathrm{SK})$ does not hold i.e. $\mathcal{D}\left(A_{1}, \ldots, A_{m}, B\right) \neq\{0\}$.

In subsection 3.1, we show that there are many situations in which (SK) does not hold, which is a motivation for the analysis developed in the following subsections. In subsection 3.2 we prove that, either $\mathcal{D}\left(A_{1}, \ldots, A_{m}, B\right)$ is a strict algebraic submanifold of $\mathbb{R}^{m}$, or, $\mathcal{D}\left(A_{1}, \ldots, A_{m}, B\right)=\mathbb{R}^{m}$. In subsection 3.3 , we prove that, in the first case, (4) is strongly stable in $L^{2}$, i. e. all $L^{2}$ solutions tend to zero in $L^{2}$ as $t \rightarrow \infty$. In subsection 3.4, we prove that, in the second one, there exist non dissipated solutions of constant $L^{2}$-norm. In subsection 3.5, we deduce a complete classification of the decomposition of the solutions in 1D $(m=1)$. In subsection 3.6 , we prove that $\mathcal{D}\left(A_{1}, \ldots, A_{m}, B\right)=\mathbb{R}^{m}$ is a necessary condition for the existence of traveling waves with $L^{2}$ profiles but this condition fails to be sufficient when $m \geqslant 2$. 


\section{$3.1 \quad(\mathrm{SK})$ is rarely satisfied}

The goal of this section is to emphasize that there are many situations in which (SK) does no hold. This fact motivates the analysis developed in the following subsections.

The first case is when $m>n$ and $B$ has form (5) with $n_{2}<n$. Indeed, in that case the set

$$
\left\{\xi \in \mathbb{R}^{m} ; \sum_{j=1}^{m} a_{k, 1}^{(j)} \xi_{j}=0 \text { for } k=2, \ldots, n\right\}
$$

is a non empty vector subspace of $\mathbb{R}^{m}$. It is contained in $\mathcal{D}\left(A_{1}, \ldots, A_{m}, B\right)$. Indeed, for every $\xi$ in this set, we have $A(\xi) e_{1}=\left(\sum_{j=1}^{m} a_{1,1}^{(j)} \xi_{j}\right) e_{1}$, thus $e_{1}$ is an eigenvector of $A(\xi)$ that belongs to $\operatorname{Ker}(B)$.

As a consequence, the property (SK) may only be satisfied when $m \leqslant n$. However, for any pair $(m, n)$ with $m \leqslant n$, and any value of $n_{1}$ there are examples for which $(S K)$ does not hold. Indeed, let $m, n \in \mathbb{N}^{*}$ with $m \leqslant n, n_{1} \in\{1, \ldots, n-$ $1\}$ and $n * n$ real matrices $A_{1}, \ldots, A_{m}, B$ with $A_{1}, \ldots, A_{m}$ symmetric and $B$ of the form (5). We assume that $a_{2,1}^{(1)} \neq 0, a_{2,1}^{(j)}=0$ for $j=2, \ldots, m$, and $a_{k, 1}^{(j)}=0$ for $j=1, \ldots, m$ and $k=3, \ldots, n$. Then $A(\xi) e_{1}=\left(\sum_{j=1}^{m} a_{1,1}^{(j)} \xi_{j}\right) e_{1}+a_{2,1}^{(1)} \xi_{1} e_{2}$. Thus, for every $\xi$ in the hyperplane

$$
\left\{\xi \in \mathbb{R}^{m} ; \xi_{1}=0\right\},
$$

$e_{1}$ is a eigenvector of $A(\xi)$ that belongs to $\operatorname{Ker}(B)$. Therefore, $\mathcal{D}\left(A_{1}, \ldots, A_{m}, B\right)$ contains this hyperplane and (SK) is not fulfilled.

\subsection{The set of degeneracy}

Proposition 2 Let $A_{1}, \ldots, A_{m}, B$ be $n * n$ real matrices such that $A_{1}, \ldots, A_{m}$ are symmetric and $B$ has form (5). The set of degeneracy $\mathcal{D}\left(A_{1}, \ldots, A_{m}, B\right)$ is an algebraic submanifold of $\mathbb{R}^{m}$. In other words, there exists a finite family of polynomials $\left(P_{j}\right)_{j \in J} \subset \mathbb{R}[X]$ such that

$$
\mathcal{D}\left(A_{1}, \ldots, A_{m}, B\right)=\left\{\xi \in \mathbb{R}^{m} ; P_{j}(\xi)=0, \forall j \in J\right\} .
$$

Thus, either $\mathcal{D}\left(A_{1}, \ldots, A_{m}, B\right)=\mathbb{R}^{m}$, or $\mathcal{D}\left(A_{1}, \ldots, A_{m}, B\right)$ has zero measure. Moreover, $\mathcal{D}\left(A_{1}, \ldots, A_{m}, B\right)$ is stable by homotheties.

Remark 6 Note that it can happen that $\mathcal{D}\left(A_{1}, \ldots, A_{m}, B\right)=\mathbb{R}^{m}$, the dissipation matrix $B$ being non-trivial. Indeed, the fact that $\mathcal{D}\left(A_{1}, \ldots, A_{m}, B\right)=\mathbb{R}^{m}$ means only that, for each $\xi$, there is a direction in which the dissipation mechanism is not effective but this is compatible, as we shall see, with $B$ being non-trivial (see Section 3.6).

Proof of Proposition 2 : Thanks to Lemma 1, one may characterize the set of degeneracy $\mathcal{D}\left(A_{1}, \ldots, A_{m}, B\right)$ as the set of those $\xi \in \mathbb{R}^{m}$ such that any $n * n$ subdeterminant of the Kalman matrix $K$ defined in (18) (with $A$ replaced by $A(\xi))$ vanishes. The polynomials $P_{j}$ in the statement of Proposition 2 are precisely those corresponding to these subdeterminants. 
Then, either all the polynomials vanish identically, and then

$$
\mathcal{D}\left(A_{1}, \ldots, A_{m}, B\right)=\mathbb{R}^{m},
$$

or, if some of them do not, the measure of $\mathcal{D}\left(A_{1}, \ldots, A_{m}, B\right)$ vanishes.

With the definition (15) of the set of degeneracy, it is clear that, when $\xi \in \mathcal{D}\left(A_{1}, \ldots, A_{m}, B\right)$ and $\alpha \in \mathbb{R}$, then, $\alpha \xi \in \mathcal{D}\left(A_{1}, \ldots, A_{m}, B\right)$.

According to the previous propositions, there are therefore only two possible cases, depending on the size of the set of degeneracy. Of course, smaller $\mathcal{D}\left(A_{1}, \ldots, A_{m}, B\right)$ is, better decay properties we expect. The case in which (SK) holds is a very particular instance where the measure of $\mathcal{D}\left(A_{1}, \ldots, A_{m}, B\right)$ vanishes, since, in that case, actually, $\mathcal{D}\left(A_{1}, \ldots, A_{m}, B\right)$ is reduced to the trivial set $\{0\}$. One of the main goals of this section is to describe what happens when the measure of the set of degeneracy vanishes, but (SK) does not hold.

To do that it is first convenient to consider some examples. The first conclusion is that, in some cases, $\mathcal{D}\left(A_{1}, \ldots, A_{m}, B\right)$ is a vector subspace. But, this is not necessarily always the case. This makes the analysis of the decay rate of (7) as a function of $\xi$ more delicate.

We introduce the notation

$$
\alpha_{k, l}=\alpha_{k, l}(\xi):=\sum_{j=1}^{m} a_{k, l}^{(j)} \xi_{j},
$$

for $\xi \in \mathbb{R}^{m}, 1 \leqslant k, l \leqslant n$, where $A_{j}=\left(a_{k, l}^{(j)}\right)_{1 \leqslant k, l \leqslant n}$. Then $A(\xi)=\left(\alpha_{k, l}\right)_{1 \leqslant k, l \leqslant n}$. Obviously, $\alpha_{k, l}=\alpha_{l, k}, 1 \leqslant k, l \leqslant n$. In the following examples, it is easier to use the definition (15) of $\mathcal{D}\left(A_{1}, \ldots, A_{m}, B\right)$ in order to compute the polynomials than using the minors of the Kalman matrix.

Example 1 : Let us consider the case $n_{1}=1$. The definition gives

$$
\mathcal{D}\left(A_{1}, \ldots, A_{m}, B\right)=\left\{\xi \in \mathbb{R}^{m} ; \alpha_{k, 1}=0, \forall k \in\{2, \ldots, n\}\right\} .
$$

Thus, $\mathcal{D}\left(A_{1}, \ldots, A_{m}, B\right)$ is a vector subspace.

Example 2 : Let us consider the case $n_{1}=2$. The definition allows characterizing $\mathcal{D}\left(A_{1}, \ldots, A_{m}, B\right)$ as the set of those $\xi \in \mathbb{R}^{m}$ for which there exists $\alpha, \beta, \gamma \in \mathbb{R}$ such that

$$
\left(\begin{array}{ll}
\alpha_{1,1} & \alpha_{1,2} \\
\alpha_{1,2} & \alpha_{2,2}
\end{array}\right)\left(\begin{array}{c}
\alpha \\
\beta
\end{array}\right)=\gamma\left(\begin{array}{c}
\alpha \\
\beta
\end{array}\right) \text { and } \alpha \alpha_{k, 1}+\beta \alpha_{k, 2}=0, \forall k \geqslant 3 .
$$

Computing explicitly $\alpha, \beta$ as functions of $\alpha_{1,1}, \alpha_{1,2}, \alpha_{2,2}$, distinguishing the cases $(\alpha, \beta)=(1,0),(\alpha, \beta)=(0,1)$ and $(\alpha \neq 0$ and $\beta \neq 0)$, we get

$$
\mathcal{D}=\mathcal{D}_{1} \cup \mathcal{D}_{2} \cup \mathcal{D}_{3+} \cup \mathcal{D}_{3-},
$$

where

$$
\mathcal{D}_{j}\left(A_{1}, \ldots A_{m}, B\right):=\left\{\xi \in \mathbb{R}^{m} ; \alpha_{k, j}=0, \forall k \neq j\right\} \text { for } j \in\{1,2\},
$$

and $\mathcal{D}_{3 \pm}\left(A_{1}, \ldots A_{m}, B\right)$ are the sets of those $\xi \in \mathbb{R}^{m}$ such that, for every $k \geqslant 3$,

$$
2 \alpha_{1,2} \alpha_{k, 1}=\left(\alpha_{1,1}-\alpha_{2,2} \pm \sqrt{\left(\alpha_{1,1}-\alpha_{2,2}\right)^{2}+4 \alpha_{1,2}^{2}}\right) \alpha_{k, 2} .
$$


Now, we distinguish two cases.

Example 2.a : Let us consider matrices $A_{1}, \ldots, A_{m}$ such that $a_{1,1}^{(j)}-a_{2,2}^{(j)}=$ $\delta a_{1,2}^{(j)}$ for $j=1, \ldots, m$, for some $\delta \in \mathbb{R}^{*}$. Then $\alpha_{1,1}-\alpha_{2,2}=\delta \alpha_{1,2}$ and

$$
\mathcal{D}_{3+} \cup \mathcal{D}_{3-}\left(A_{1}, \ldots A_{m}, B\right)=\left\{\xi \in \mathbb{R}^{m} ; 2 \alpha_{k, 1}=\left[\delta \pm \sqrt{\delta^{2}+4}\right] \alpha_{k, 2}, \forall 3 \leqslant k \leqslant n\right\} .
$$

Thus $\mathcal{D}\left(A_{1}, \ldots, A_{m}, B\right)$ is the union of a finite number of vector subspaces of $\mathbb{R}^{m}$.

Example 2.b : Now, let us take $n=3, m=5$ and $A_{1}, \ldots, A_{5}$ defined by

$$
\begin{array}{ll}
A_{1}:=\left(\begin{array}{lll}
1 & 0 & 0 \\
0 & 0 & 0 \\
0 & 0 & *
\end{array}\right), A_{2}:=\left(\begin{array}{lll}
0 & 0 & 0 \\
0 & 1 & 0 \\
0 & 0 & *
\end{array}\right), A_{3}:=\left(\begin{array}{lll}
0 & 1 & 0 \\
1 & 0 & 0 \\
0 & 0 & *
\end{array}\right), \\
A_{4}:=\left(\begin{array}{lll}
0 & 0 & 1 \\
0 & 0 & 0 \\
1 & 0 & *
\end{array}\right), A_{5}:=\left(\begin{array}{lll}
0 & 0 & 0 \\
0 & 0 & 1 \\
0 & 1 & *
\end{array}\right),
\end{array}
$$

where the notation $*$ means that the corresponding coefficient may have any real value. Then, $\alpha_{1,1}=\xi_{1}, \alpha_{2,2}=\xi_{2}, \alpha_{1,2}=\xi_{3}, \alpha_{3,1}=\xi_{4}, \alpha_{3,2}=\xi_{5}$. Thus

$$
\mathcal{D}_{3 \pm}\left(A_{1}, \ldots A_{5}, B\right)=\left\{\xi \in \mathbb{R}^{5} ; 2 \xi_{3} \xi_{4}+\left(\xi_{2}-\xi_{1}\right) \xi_{5}= \pm \xi_{5} \sqrt{\left(\xi_{1}-\xi_{2}\right)^{2}+4 \xi_{3}^{2}}\right\} .
$$

Obviously, $\mathcal{D}_{3 \pm}$ are not vector subspaces and, consequently, the set of degeneracy can not be written as the union of a finite number of vector subspaces of $\mathbb{R}^{m}$ neither.

\subsection{A NSC for strong $L^{2}$ stability}

Proposition 3 Let $A_{1}, \ldots, A_{m}, B$ be $n * n$ real matrices with $B$ of the form (5), $A_{1}, \ldots, A_{m}$ being symmetric. The following statements are equivalent

- (1) the measure of $\mathcal{D}\left(A_{1}, \ldots, A_{m}, B\right)$ vanishes,

- (2) any solution of (4) with $L^{2}\left(\mathbb{R}^{m}, \mathbb{R}^{n}\right)$ initial condition converges to zero strongly in $L^{2}\left(\mathbb{R}^{m}, \mathbb{R}^{n}\right)$ as $t \rightarrow \infty$.

Proof: First, we prove that "(1) $\Rightarrow(\mathbf{2})$ ". We assume that the measure of $\mathcal{D}\left(A_{1}, \ldots, A_{m}, B\right)$ vanishes. Let $w_{0} \in L^{2}\left(\mathbb{R}^{m}, \mathbb{R}^{n}\right)$ and $w$ be the solution of (4) with initial condition $w_{0}$. Applying Proposition 1, we get, for $\epsilon>0$ small enough,

$$
|\hat{w}(t, \xi)| \leqslant \sqrt{3}\left|\hat{w}_{0}(\xi)\right| e^{-\tilde{c} \min \left\{|\xi|^{2}, 1\right\} N_{*}(\epsilon) t}
$$

where $\omega:=\xi /|\xi|$. Thanks to the implication "(1) $\Rightarrow(\mathbf{4})$ " in Lemma 1 and since the measure of $\mathcal{D}\left(A_{1}, \ldots, A_{m}, B\right)$ vanishes, then, for almost every $\omega \in S^{m-1}$, $N_{*, \epsilon}(\omega)>0$. Thus $\hat{w}(t, \xi) \rightarrow 0$ when $t \rightarrow+\infty$ for almost every $\xi \in \mathbb{R}^{m}$. Moreover, $|\hat{w}(t,).| \leqslant\left|\hat{w}_{0}().\right| \in L^{2}\left(\mathbb{R}^{m}, \mathbb{R}^{n}\right)$. Thus, the dominated convergence theorem implies that $\hat{w}(t)$ converges to zero in $L^{2}\left(\mathbb{R}^{m}, \mathbb{R}^{n}\right)$ when $t \rightarrow+\infty$.

Now, we prove "(2) $\Rightarrow(\mathbf{1})$ ". Let us assume (2) holds. Let $\varphi \in \mathcal{S}\left(\mathbb{R}^{m}, \mathbb{R}\right)$ be such that $\varphi>0$ on $\mathbb{R}^{m}$. Let $w_{0}^{(1)} \in L^{2}\left(\mathbb{R}^{m}, \mathbb{R}^{n}\right)$ be defined by $\hat{w}_{0}^{(1)}(\xi):=\varphi(\xi) e_{1}$ and $w^{(1)}$ be the solution of (4) with initial condition $w_{0}^{(1)}$. One has

$$
\hat{w}^{(1)}(t, \xi)=\exp [E(\xi) t] e_{1} \varphi(\xi) .
$$


Since $w^{(1)}(t) \rightarrow 0$ strongly in $L^{2}\left(\mathbb{R}^{m}, \mathbb{R}^{n}\right)$, there exists an increasing sequence $\left(t_{p}^{(1)}\right)_{p \in \mathbb{N}} \subset \mathbb{R}_{+}$such that

$$
\exp \left[E(\xi) t_{p}^{(1)}\right] e_{1} \rightarrow 0 \text { when }[p \rightarrow+\infty] \text { for almost every } \xi \in \mathbb{R}^{m} .
$$

Let $w_{0}^{(2)} \in L^{2}\left(\mathbb{R}^{m}, \mathbb{R}^{n}\right)$ be defined by $\hat{w}_{0}^{(2)}(\xi):=\varphi(\xi) e_{2}$ and $w^{(2)}$ be the solution of (4) with initial condition $w_{0}^{(2)}$. The sequence $\left(w^{(2)}\left(t_{p}^{(1)}\right)\right)_{p \in \mathbb{N}}$ tends to zero strongly in $L^{2}\left(\mathbb{R}^{m}, \mathbb{R}^{n}\right)$. Thus, there exists a subsequence $\left(t_{p}^{(2)}\right)_{p \in \mathbb{N}}$ of $\left(t_{p}^{(1)}\right)_{p \in \mathbb{N}}$ such that

$$
\exp \left[E(\xi) t_{p}^{(2)}\right] e_{2} \rightarrow 0 \text { when }[p \rightarrow+\infty] \text { for almost every } \xi \in \mathbb{R}^{m} \text {. }
$$

Iterating this process for $k=1, \ldots, n$, one gets an increasing sequence $\left(t_{p}\right)_{p \in \mathbb{N}} \subset$ $\mathbb{R}_{+}$and a subset $\mathcal{N}$ of $\mathbb{R}^{m}$ of zero measure such that

$$
\exp \left[E(\xi) t_{p}\right] \rightarrow 0 \text { in } \mathbb{R}^{n * n}, \forall \xi \in \mathbb{R}^{m}-\mathcal{N} .
$$

Let $\xi \in \mathbb{R}^{m}-\mathcal{N}$. The convergence (42) implies that the real part of any eigenvalue of $E(\xi)$ has a negative real part. Thus, $A(\xi)$ has no eigenvector in $\operatorname{Ker}(B)$ since, otherwise, one would get an eigenvalue $\lambda$ of $E(\xi)$ with $\Re(\lambda)=0$. In conclusion $\mathcal{D}\left(A_{1}, \ldots, A_{m}, B\right)$ is contained in $\mathcal{N}$ and, consequently, its measure vanishes.

Remark 7 As a consequence of this result, and in view of Proposition 2 which classifies the possible structures for the set of degeneracy, we deduce that, whenever $\mathcal{D}\left(A_{1}, \ldots, A_{m}, B\right)$ is a strict subset of $\mathbb{R}^{m}$, all solutions tend to zero in $L^{2}$ strongly as $t \rightarrow \infty$. In the following section we show that, in the other case, $i$. e. when $\mathcal{D}\left(A_{1}, \ldots, A_{m}, B\right)$ coincides with $\mathbb{R}^{m}$, there are non-trivial solutions of constant $L^{2}$-norm.

\subsection{A NSC for the existence of non dissipated solutions}

Proposition 4 Let $A_{1}, \ldots, A_{m}, B$ be $n * n$ real matrices with $B$ of the form (5) and $A_{1}, \ldots, A_{m}$ symmetric. The following statements are equivalent

- (1) $\mathcal{D}\left(A_{1}, \ldots, A_{m}, B\right)=\mathbb{R}^{m}$,

- (2) there exists a non dissipated solution of (4), i.e. with a constant $L^{2}$ norm.

The following Lemma will be important in the proof of Proposition 4.

Lemma 2 Let $A_{1}, \ldots, A_{m}, B$ be $n * n$ real matrices with $B$ of the form (5) and $A_{1}, \ldots, A_{m}$ symmetric. Let $w^{0} \in L^{2}\left(\mathbb{R}^{m}, \mathbb{R}^{n}\right)$ and $w$ be the solution of (4) with initial condition $w^{0}$. The following statements are equivalent

- (1) $w$ is not dissipated i.e. $\|w(t)\|_{L^{2}} \equiv\left\|w^{0}\right\|_{L^{2}}$

- (2) for almost every $\xi \in \mathbb{R}^{m}, \hat{w}^{0}(\xi)$ belongs to

$$
V(\xi):=\left\{v \in \mathbb{C}^{n}, B A(\xi)^{k} v=0, \forall k \in \mathbb{N}\right\}
$$


Proof of Lemma 2 : $\quad$ Let us prove $(\mathbf{1}) \Rightarrow(\mathbf{2})$. We assume $w$ is not dissipated. We have

$$
\frac{d}{d t} \int_{\mathbb{R}^{m}}|w(t, x)|^{2} d x=-2 \int_{\mathbb{R}^{m}}\langle B w(t, x), w(t, x)\rangle d x \equiv 0,
$$

thus $B w \equiv 0$, which implies $B \hat{w} \equiv 0$. Thus the expression (9) can be written

$$
\hat{w}(t, \xi)=\exp [-i A(\xi) t] \hat{w}^{0}(\xi)
$$

The equality $B \hat{w} \equiv 0$ implies

$$
B \exp [-i A(\xi) t] \hat{w}^{0}(\xi) \text {, for every } t \in \mathbb{R}_{+} \text {, for almost every } \xi \in \mathbb{R}^{m} .
$$

Differentiating $k$ times this equality with respect to time at $t=0$, we get (2).

Now, let us prove $(\mathbf{2}) \Rightarrow(\mathbf{1})$. We assume that, for almost every $\xi \in \mathbb{R}^{m}$, $\hat{w}_{0}(\xi) \in V(\xi)$. By definition, $V(\xi)$ is a vector subspace of $\operatorname{Ker}(B)$ stable by $A(\xi)$, thus $(9)$ gives

$\hat{w}(t, \xi)=\sum_{k=0}^{\infty} \frac{t^{k}}{k !}[-B-i A(\xi)]^{k} \hat{w}^{0}(\xi)=\sum_{k=0}^{\infty} \frac{t^{k}}{k !}[-i A(\xi)]^{k} \hat{w}^{0}(\xi)=\exp [-i A(\xi) t] \hat{w}^{0}(\xi)$.

Therefore $\|w(t)\|_{L^{2}}=\|\hat{w}(t)\|_{L^{2}} \equiv\left\|w_{0}\right\|_{L^{2}}$, i.e. (1) holds.

Proof of Proposition 4 : Let us prove $(\mathbf{2}) \Rightarrow(\mathbf{1})$. We assume there exists a non dissipated solution $w$, associated to an initial condition $w^{0} \in L^{2}\left(\mathbb{R}^{m}, \mathbb{R}^{n}\right)$ with $w^{0} \neq 0$. Thanks to Lemma 2 , we know that, for almost every $\xi \in \mathbb{R}^{m}$, $\hat{w}^{0}(\xi) \in V(\xi)$. So, for almost every $\xi \in \operatorname{Supp}\left(w_{0}\right)$ (the support of $\left.w_{0}\right), V(\xi)$ is a non empty vector subspace of $\operatorname{Ker}(B)$ stable by $A(\xi)$, moreover $\left.A(\xi)\right|_{V(\xi)}$ is symmetric, thus it has an eigenvector. We have proved that $\operatorname{Supp}\left(w^{0}\right) \subset$ $\mathcal{D}\left(A_{1}, \ldots, A_{m}, B\right)$, thus the measure of $\mathcal{D}\left(A_{1}, \ldots, A_{m}, B\right)$ does not vanish and Proposition 2 implies that (1) holds.

Now, let us prove $(\mathbf{1}) \Rightarrow(\mathbf{2})$. We assume $\mathcal{D}\left(A_{1}, \ldots, A_{m}, B\right)=\mathbb{R}^{m}$. Then for every $\xi \in \mathbb{R}^{m}, V(\xi) \neq\{0\}$ because $V(\xi)$ contains at least an eigenvector of $A(\xi)$ that belongs to $\operatorname{Ker}(B)$. In order to prove that (2) holds, we build an $L^{2}\left(\mathbb{R}^{m}, \mathbb{R}^{n}\right)$ initial condition $w^{0}$ such that $\hat{w}^{0}(\xi) \in V(\xi)$, for every $\xi \in \mathbb{R}^{m}$, which gives the conclusion thanks to Lemma 2 .

The Cayley-Hamilton theorem justifies that

$$
V(\xi)=\left\{v \in \mathbb{C}^{n} ; B A(\xi)^{k} v=0 \text { for } k=0, \ldots, n-1\right\}=\operatorname{Ker}\left(M_{1}(\xi)\right),
$$

where $M_{1}(\xi)=\sum_{k=0}^{n-1} A(\xi)^{k} B^{t} B A(\xi)^{k}$ depends polynomially in $\xi$. For $\xi \in \mathbb{R}^{m}$, let $P(\xi)$ be the orthogonal projection $\mathbb{R}^{n} \rightarrow \operatorname{Ker}\left(M_{1}(\xi)\right.$ ). Let $\xi^{*} \in \mathbb{R}^{m}$ and $z^{*} \in S^{n-1} \cap \operatorname{Ker}\left(M_{1}\left(\xi^{*}\right)\right)$. The map $\xi \in \mathbb{R}^{m} \mapsto P(\xi) z^{*}$ is continuous at $\xi^{*}$ because $M_{1}(\xi)$ is an analytic perturbation of $M_{1}\left(\xi^{*}\right)$ (see, for example [12, Chapter 2, Section 1.4]). Thus, there exists a neighborhood $\Omega_{1}$ of $\xi^{*}$ in $\mathbb{R}^{m}$ with a positive finite measure such that $\left\|P(\xi) z^{*}\right\|>1 / 2$, for every $\xi \in \Omega_{1}$. We define

$$
\hat{w}^{0}(\xi):=P(\xi) z^{*} 1_{\Omega_{1} \cup\left(-\Omega_{1}\right)}(\xi) .
$$

Then $w^{0} \in L^{2}\left(\mathbb{R}^{m}, \mathbb{R}^{n}\right)$ because $\left|\hat{w}^{0}(\xi)\right| \leqslant 1_{\Omega_{1} \cup\left(-\Omega_{1}\right)}(\xi)$ and $\hat{w}^{0}(-\xi)=\overline{\hat{w}^{0}(\xi)}$. Moreover, $w^{0} \neq 0$ because $\left\|\hat{w}^{0}(\xi)\right\| \geqslant 1 / 2, \forall \xi \in \Omega_{1} \cup\left(-\Omega_{1}\right)$. 
Remark 8 According to the results in this section and the previous one, we deduce that there are only two possibilities for the set of degeneracy which lead to different asymptotic properties as $t \rightarrow \infty$ :

- Whenever $\mathcal{D}\left(A_{1}, \ldots, A_{m}, B\right)$ is a strict subset of $\mathbb{R}^{m}$, its measure vanishes, and all solutions tend to zero in $L^{2}$ strongly as $t \rightarrow \infty$.

- When $\mathcal{D}\left(A_{1}, \ldots, A_{m}, B\right)$ coincides with $\mathbb{R}^{m}$, there are non-trivial solutions of constant $L^{2}$-norm.

\subsection{Complete classification in the case $m=1$}

In this section we consider the $1 \mathrm{D}$ problem

$$
w_{t}+A w_{x}=B w
$$

where $A, B \in \mathbb{R}^{n * n}, w \in \mathbb{R}^{n}$ and $x \in \mathbb{R}$. Note that this simple case can be treated directly and without the analysis developed in the previous subsections. However, it is clarifying to treat it in the framework of the general theory we have developed.

When $m=1, A(\xi)=\xi A$, since $\xi$ is now scalar. Therefore, the (SK) condition does not involve the Fourier variable $\xi$ and can be written simply as

$$
\text { (SK) : } \operatorname{Ker}(B) \cap\{\text { eigenvectors of } A\}=\{0\} \text {. }
$$

Obviously, this is equivalent to requiring that the pair $(A, B)$ satisfies the Kalman rank condition

In $1 \mathrm{D}(m=1)$, the $(\mathrm{SK})$ condition characterizes completely the asymptotic behavior of the solutions of (43).

Proposition 5 We assume that $A$ is symmetric and $B$ has the form (5). The following holds:

- The following statements are equivalent

- (1) $A$ and $B$ satisfy $(S K):\{$ eigenvectors of $A\} \cap \operatorname{Ker}(B)=\{0\}$,

- (2) there exist $C, \lambda>0$ such that, for every $w^{0} \in L^{1} \cap L^{2}\left(\mathbb{R}, \mathbb{R}^{n}\right)$, the solution of (43) can be decomposed as $w=w_{1}+w_{2}$ where (14) holds with $m=1$.

- Moreover, if (SK) is not satisfied, any solution of (43) with initial condition in $L^{2}\left(\mathbb{R}, \mathbb{R}^{n}\right)$ may be decomposed as $w=w_{1}+w_{2}+w_{\text {trw }}$ where $w_{1}$, $w_{2}$ satisfy the previous estimates and $w_{\text {trw }}$ is a pure transport term constituted by a finite sum of traveling waves, $w_{t r w}(t, x)=\sum_{j=1}^{r} w_{t r w, j}\left(x-\lambda_{j} t\right)$, where $r \leqslant n, \lambda_{j} \in \mathbb{R}$ and $w_{\text {trw }, j} \in L^{2}\left(\mathbb{R}, \mathbb{R}^{n}\right)$ for $j=0, \ldots, r$.

Proof of Proposition 5 : First, the implication " $(\mathbf{1}) \Rightarrow(\mathbf{2})$ " was proved in Theorem 1.

Now, we prove the second statement, that also gives " $(\mathbf{2}) \Rightarrow(\mathbf{1})$ ".

Assume that (SK) is false. Let $V$ be the subspace of $\operatorname{Ker}(B)$ which is stable by $A(A V \subset V)$ with maximal dimension constituted by the sum of all subspaces 
of $\operatorname{Ker}(B)$ stable by $A$. Let $r:=\operatorname{dim}(V)$. The endomorphism $\left.A\right|_{V}$ is symmetric thus, there exists an orthonormal basis $\left(v_{1}, \ldots, v_{r}\right)$ of $V$ made of eigenvectors of $A$ : $A v_{j}=\lambda_{j} v_{j}, j=1, \ldots, r$. Let $V^{\perp}$ be the orthogonal supplementary of $V$ in $\operatorname{Ker}(B)$ (i.e. $\left.\operatorname{Ker}(B)=V+V^{\perp}\right)$ and $\left(v_{r+1}, \ldots, v_{n_{1}}\right)$ be an orthonormal basis of $V^{\perp}$. Then, $\left(v_{1}, \ldots, v_{n_{1}}\right)$ is an orthonormal basis of $\operatorname{Ker}(B)$. Since $\operatorname{Ker}(B)=$ $\operatorname{Span}\left(e_{1}, \ldots, e_{n_{1}}\right)$, then $\mathcal{V}:=\left(v_{1}, \ldots, v_{n_{1}}, e_{n_{1}+1}, \ldots, e_{n}\right)$ is an orthonormal basis of $\mathbb{R}^{n}$. Let $P$ be the basis change matrix from the canonical basis $\left(e_{1}, \ldots, e_{n}\right)$ to the basis $\mathcal{V}$ (the columns of $P$ are the components of the vectors of $\mathcal{V}$ in the canonical basis $\left.\left(e_{1}, \ldots, e_{n}\right)\right)$. Then $P^{-1} B P=B$,

$$
\begin{aligned}
& \left\langle A v_{j}, v_{i}\right\rangle=\left\langle v_{j}, A v_{i}\right\rangle=\lambda_{i}\left\langle v_{j}, v_{i}\right\rangle=0, \forall j \in\left\{r+1, \ldots, n_{1}\right\}, \forall i \in\{1, \ldots, r\}, \\
& \left\langle A e_{j}, v_{i}\right\rangle=\left\langle e_{j}, A v_{i}\right\rangle=\lambda_{i}\left\langle e_{j}, v_{i}\right\rangle=0, \forall j \in\left\{n_{1}+1, \ldots, n\right\}, \forall i \in\{1, \ldots, r\} .
\end{aligned}
$$

Thus

$$
\tilde{A}:=P^{-1} A P=\left(\begin{array}{ccc|ccc|ccc}
\lambda_{1} & & & 0 & \ldots & 0 & 0 & \ldots & 0 \\
& \ldots & & \ldots & \ldots & \ldots & \ldots & \ldots & \ldots \\
& & \lambda_{r} & 0 & \ldots & 0 & 0 & \ldots & 0 \\
\hline 0 & \ldots & 0 & * & \ldots & * & * & \ldots & * \\
0 & \ldots & 0 & * & \ldots & * & * & \ldots & * \\
0 & \ldots & 0 & * & \ldots & * & * & \ldots & * \\
\hline 0 & \ldots & 0 & * & \ldots & * & * & \ldots & * \\
0 & \ldots & 0 & * & \ldots & * & * & \ldots & * \\
0 & \ldots & 0 & * & \ldots & * & * & \ldots & *
\end{array}\right)
$$

where the first diagonal block is of dimension $r * r$, the second one is of dimension $\left(n_{1}-r\right) *\left(n_{1}-r\right)$ and the third one is of dimension $n_{2} * n_{2}$. Then $\tilde{w}:=P^{-1} w$ solves

$$
\tilde{w}_{t}+\tilde{A} \tilde{w}_{x}=-B \tilde{w}
$$

and $\tilde{w}_{j}(t, x)=\tilde{w}_{j}\left(0, x-\lambda_{j} t\right)$ for $j=1, \ldots, r$. These components are pure transport terms. We define

$$
w_{t r w}:=P\left(\begin{array}{c}
\tilde{w}_{1} \\
\ldots \\
\tilde{w}_{r} \\
0 \\
\ldots \\
0
\end{array}\right)
$$

The $(n-r)$ latest components of $\tilde{w}$ solve an hyperbolic system fulfilling the (SK) condition. Thus, it may be decomposed as $\tilde{w}_{1}+\tilde{w}_{2}$ with (14) for $m=1$. Finally, we take $w_{1}:=P \tilde{w}_{1}$ and $w_{2}:=P \tilde{w}_{2}$. This completes the decomposition in three terms of the second statement.

Note that this argument shows that " $(2) \Rightarrow(\mathbf{1})$ ", as claimed. Indeed, if (1) is not satisfied, i. e. the (SK) is not fulfilled, the argument above shows that, then, there is a traveling wave component that does not decay, thus showing that (2) does not hold either.

\subsection{The case $\mathcal{D}\left(A_{1}, \ldots, A_{m}, B\right)=\mathbb{R}^{m}$ : traveling waves?}

Obviously, the case $\mathcal{D}\left(A_{1}, \ldots, A_{m}, B\right)=\mathbb{R}^{m}$ is the most degenerate one. Proposition 4 shows that, in this case, there exist non dissipated solutions. In $1 \mathrm{D}$ 
$(m=1)$, any non dissipated solution is a finite sum of traveling waves (see Proposition 5). Thus, it is natural to ask whether this is also true for $m \geqslant 2$. As we shall see, this is not the case.

Proposition 6 We assume $m \geqslant 2$. The equality $\mathcal{D}\left(A_{1}, \ldots, A_{m}, B\right)=\mathbb{R}^{m}$ is necessary for the existence of a non trivial traveling wave solution of (4) with an $L^{2}\left(\mathbb{R}^{m}, \mathbb{R}^{n}\right)$-profile but it is not sufficient.

Remark 9 As a consequence, when $m \geqslant 2$, the non dissipated solutions exhibit more complex structures that being sums of traveling waves.

Proof : $\quad$ First, we prove that $\mathcal{D}\left(A_{1}, \ldots, A_{m}, B\right)=\mathbb{R}^{m}$ is necessary for the existence of traveling waves solutions. Indeed, any non trivial traveling wave solution is a non dissipated solution. Thus Proposition 4 gives the result.

Now, we prove that $\mathcal{D}\left(A_{1}, \ldots, A_{m}, B\right)=\mathbb{R}^{m}$ is not sufficient for the existence of traveling waves.

Let us remark that, when (4) has a traveling wave solution, then there exists $c \in \mathbb{R}^{m}$ and $\Omega \subset \mathbb{R}^{m}$ with positive measure, such that, for almost every $\xi \in \Omega$, $A(\xi)$ has an eigenvalue $\lambda(\xi)$ of the form

$$
\lambda(\xi)=\sum_{j=1}^{m} c_{j} \xi_{j}
$$

associated to an eigenvector $v(\xi)$ that belongs to $\operatorname{Ker}(B)$. As we will see, one can easily build examples of matrices $A_{1}, \ldots, A_{m}, B$ for which the later is impossible, but $\mathcal{D}\left(A_{1}, \ldots, A_{m}, B\right)=\mathbb{R}^{m}$

We consider the system (4) with $m=2$ and

$$
A_{1}:=\left(\begin{array}{ccc}
a_{1} & a_{1,2} & * \\
a_{1,2} & a_{2} & * \\
0 & 0 & *
\end{array}\right), \quad A_{2}:=\left(\begin{array}{ccc}
c_{1} & c_{1,2} & * \\
c_{1,2} & c_{2} & * \\
0 & 0 & *
\end{array}\right), \quad B:=\left(\begin{array}{ccc}
0 & 0 & 0 \\
0 & 0 & 0 \\
0 & 0 & 1
\end{array}\right)
$$

with $a_{1,2} \neq 0, c_{1,2} \neq 0$ and

$$
\begin{gathered}
\frac{\delta_{a}}{a_{1,2}} \neq \frac{\delta_{c}}{c_{1,2}}, \\
4 \delta_{a} \delta_{c} a_{1,2} c_{1,2} \neq\left(\delta_{a} c_{1,2}\right)^{2}+\left(\delta_{c} a_{1,2}\right)^{2},
\end{gathered}
$$

where $\delta_{a}:=a_{2}-a_{1}$ and $\delta_{c}:=c_{2}-c_{1}$. The notation "*" means that the corresponding coefficient may have any value. We claim that, for this choice of the parameters $a_{1}, a_{1,2}, a_{2}, c_{1}, c_{1,2}, c_{2}$, one has $\mathcal{D}\left(A_{1}, A_{2}, B\right)=\mathbb{R}^{2}$ but the eigenvalues of $\left.\left(\xi_{1} A_{1}+\xi_{2} A_{2}\right)\right|_{\operatorname{Ker}(B)}$ cannot have the form (44) on a subset of $\mathbb{R}^{2}$ with positive measure.

For $\left(\xi_{1}, \xi_{2}\right) \in \mathbb{R}^{2}-\{0\}$, the subspace $\operatorname{Ker}(B)$ is stable by $A(\xi)=\xi_{1} A_{1}+$ $\xi_{2} A_{2}$, and the restriction $\left.A(\xi)\right|_{\operatorname{Ker}(B)}$ is symmetric. Thus it has an eigenvector. Therefore $\mathcal{D}\left(A_{1}, A_{2}, B\right)=\mathbb{R}^{2}$.

The eigenvalues of $\left.\left(\xi_{1} A_{1}+\xi_{2} A_{2}\right)\right|_{\operatorname{Ker}(B)}$ are

$$
\lambda_{ \pm}\left(\xi_{1}, \xi_{2}\right):=\frac{1}{2}\left(\left(a_{1}+a_{2}\right) \xi_{1}+\left(c_{1}+c_{2}\right) \xi_{2} \pm \sqrt{\Delta\left(\xi_{1}, \xi_{2}\right)}\right)
$$


where

$$
\Delta\left(\xi_{1}, \xi_{2}\right)=\left[\delta_{a} \xi_{1}+\delta_{c} \xi_{2}\right]^{2}+4\left[a_{1,2} \xi_{1}+c_{1,2} \xi_{2}\right]^{2} .
$$

Thanks to (45), we have $\Delta\left(\xi_{1}, \xi_{2}\right)>0$ and there does not exist any $\alpha, \beta \in \mathbb{R}$ such that

$$
\Delta\left(\xi_{1}, \xi_{2}\right)=\left(\alpha \xi_{1}+\beta \xi_{2}\right)^{2}
$$

on a subset of $\mathbb{R}^{m}$ with positive measure because of (46). Thus, non trivial traveling wave solutions may not exist.

Remark 10 The structure of the non dissipated solutions in a general context needs to be further investigated.

\section{Decomposition of the solutions when $\mathcal{D}$ is the union of vector subspaces}

As proved in previous sections, whenever the measure of $\mathcal{D}\left(A_{1}, \ldots, A_{m}, B\right)$ vanishes, all solutions tend to zero in $L^{2}$ as $t \rightarrow \infty$. This section is devoted to analyze the asymptotic behavior in some more detail. As emphasized in Remark 1, the asymptotic behavior of the solutions of (4) reduces to the study of the real valued function $\omega \in S^{m-1} \mapsto N_{*, \epsilon}(\omega)$, defined in Proposition 1. This study is the key point of this section.

In all this section, we will consider situations in which the set of degeneracy $\mathcal{D}\left(A_{1}, \ldots, A_{m}, B\right)$ has zero measure and is the union of vector subspaces. This assumption is restrictive because, in general, when the measure of $\mathcal{D}\left(A_{1}, \ldots, A_{m}, B\right)$ vanishes, this set is an algebraic submanifold (see Proposition 2), which is not necessarily a union of vector subspaces (see examples 1, 2.a, 2.b in subsection 3.2). However, this assumption holds in many particular examples, studied in this section.

In subsection 4.1, we state a decomposition for the solutions of (4) when $\mathcal{D}\left(A_{1}, \ldots, A_{m}, B\right)$ is a union of vector subspaces and under the additional assumption

$$
N_{*, \epsilon}(\omega) \geqslant c \operatorname{dist}\left(\omega, S^{m-1} \cap \mathcal{D}\right)^{\alpha}, \forall \omega \in S^{m-1}
$$

for some $\alpha \geqslant 2, c>0$ (see Proposition 7).

In subsection 4.2, we prove that, in the particular case $n_{1}=1$ (i.e. $\operatorname{Ker}(B)=$ $\left.\operatorname{Span}\left(e_{1}\right)\right), \mathcal{D}\left(A_{1}, \ldots, A_{m}, B\right)$ is a vector subspace of $\mathbb{R}^{m}$ and (47) holds with $\alpha=2$ (see Proposition 8). This leads to a decomposition of the solutions of (4) with $n_{1}=1$ (see Theorem 2).

In subsection 4.3 , we consider different explicit examples, in which the degenerate set $\mathcal{D}\left(A_{1}, \ldots, A_{m}, B\right)$ is a union of vector subspaces and the smallest exponent in (47) is $\alpha=2$ or $\alpha=4$. We deduce a decomposition of the solutions of (4) for these particular cases (see Theorem 3). With these examples, we see that, when $m \geqslant 2$, there is a whole class of phenomena that do not arise under (SK).

Finally, in subsection 4.4, we write a complete classification of the different possible asymptotic behaviors when $n=2$. 


\subsection{General statement}

For $g: \mathbb{R}^{m} \rightarrow \mathbb{R}^{n}$, we define $L(g):(0,+\infty) \rightarrow(0,+\infty]$,

$$
L(g)(\rho):=\sup \left\{|g(\xi)| ; \xi \in \mathbb{R}^{m},|\xi|=\rho\right\} .
$$

Proposition 7 We assume $m \geqslant 2$. Let $A_{1}, \ldots, A_{m}, B$ be $n * n$ real matrices with $A_{1}, \ldots, A_{m}$ symmetric and $B$ of the form (5). Let $\epsilon \in\left(0, \epsilon_{*}\right)$ where $\epsilon_{*}$ is as in Proposition 1. We assume

- (A1)

$$
\mathcal{D}\left(A_{1}, \ldots, A_{m}, B\right)=\cup_{j=1}^{J} \mathcal{D}_{j},
$$

where $J \in \mathbb{N}^{*}$ and $\mathcal{D}_{j}$ is a vector subspace of $\mathbb{R}^{m}$ with codimension $r_{j} \in \mathbb{N}^{*}$,

- (A2) there exists $c_{j}>0, \alpha_{j} \geqslant 2$ for $j=1, \ldots, J$, such that, for every $\omega \in S^{m-1}$ with $\operatorname{dist}\left(\omega, \mathcal{D}\left(A_{1}, \ldots, A_{m}, B\right)\right)=\operatorname{dist}\left(\omega, \mathcal{D}_{j}\right)$,

$$
N_{*, \epsilon}(\omega) \geqslant c_{j} \operatorname{dist}\left(\omega, \mathcal{D}_{j}\right)^{\alpha_{j}} .
$$

Then, there exists $C=C\left(A_{1}, \ldots, A_{m}, B\right)$ and $\lambda=\lambda\left(A_{1}, \ldots, A_{m}, B\right)>0$ such that, for every $w^{0} \in L^{1} \cap L^{2}\left(\mathbb{R}^{m}, \mathbb{R}^{n}\right)$ with

$$
N_{1}\left(w^{0}\right):=\int_{1}^{\infty} \rho^{m-1} L\left[\hat{w}^{0}\right](\rho) d \rho<\infty,
$$

the solution of (4) with initial condition $w^{0}$ can be decomposed as

$$
w=w_{1}+w_{2}+w_{3}+w_{4}
$$

where (14) holds and

$$
\left\|w_{3}(t)\right\|_{L^{\infty}\left(\mathbb{R}^{m}, \mathbb{R}^{n}\right)} \leqslant \frac{C}{t^{\frac{1}{\alpha}}} N_{1}\left(w^{0}\right), \forall t \in(0,+\infty),
$$

where $\alpha:=\max \left\{\alpha_{1}, \ldots, \alpha_{J}\right\}$ and

$$
\left\|w_{4}(t)\right\|_{L^{\infty}\left(\mathbb{R}^{m}, \mathbb{R}^{n}\right)} \leqslant C\left(\sum_{j=1}^{J} \frac{1}{t^{\frac{r_{j}}{\alpha_{j}}}}\right)\left\|w^{0}\right\|_{L^{1}\left(\mathbb{R}^{m}, \mathbb{R}^{n}\right)}, \forall t \in(0,+\infty) .
$$

Remark 11 The assumption (49) holds, in particular, when $w^{0}$ belongs to the the Schwartz space $\mathcal{S}\left(\mathbb{R}^{m}, \mathbb{R}^{n}\right)$. Indeed, in that case, $\hat{w}^{0} \in \mathcal{S}\left(\mathbb{R}^{m}, \mathbb{R}^{n}\right)$, Thus, there exists $C>0$ such that, for every $\xi \in \mathbb{R}^{m}$,

$$
\left|\hat{w}^{0}(\xi)\right| \leqslant \frac{C}{|\xi|^{m+1}},
$$

which leads to

$$
\int_{1}^{\infty} \rho^{m-1} L\left(\hat{w}^{0}\right)(\rho) d \rho \leqslant \int_{1}^{\infty} \frac{C}{\rho^{2}} d \rho .
$$

The assumption (49) also holds when $w^{0}$ belongs to $H^{s}\left(\mathbb{R}^{m}, \mathbb{R}^{n}\right)$ with s large enough. 
Remark 12 We refer to the Examples 1 and 2.a of Subsection 3.2 for which the assumption $(\boldsymbol{A} 1)$ holds with different values for the codimensions $r_{j}$. For example, in the example 2.a, any $\mathcal{D}_{j}$ is defined by $(n-2)$ linear equations, but some of them may be linearly dependent, thus the codimension of $\mathcal{D}_{j}$ may take any value $r_{j} \in\{1, \ldots, m-1\}$ by varying $n$ and the coefficients of the matrices $A_{1}, \ldots, A_{m}$.

Note that the assumption $\alpha_{j} \geqslant 2$ is natural. Indeed, let us consider $\omega^{*} \in$ $S^{m-1} \cap \mathcal{D}\left(A_{1}, \ldots, A_{m}, B\right)$ and let us assume that the map $\omega \mapsto N_{*, \epsilon}(\omega)$ is smooth in a neighborhood of $\omega^{*}$ (this can be justified, for example, when $N_{*, \epsilon}\left(\omega^{*}\right)=0$ is a simple eigenvalue of the matrix $M_{\epsilon}\left(\omega^{*}\right)$ defined by (23)). Since $N_{*, \epsilon}$ is non negative and $N_{*, \epsilon}\left(\omega^{*}\right)=0$, then, necessarily, $d N_{*, \epsilon}\left(\omega^{*}\right)=0$. Thus, the Taylor formula justifies that, in a neighborhood of $\omega^{*}$ we have

$$
N_{*, \epsilon}(\omega) \leqslant\left|d^{2} N_{*, \epsilon}\left(\omega^{*}\right)\right|\left|\omega-\omega^{*}\right|^{2} .
$$

Therefore, if (A2) holds, then, necessarily $\alpha_{j} \geqslant 2$.

Remark 13 Note that in Proposition 7, we do not assume (SK) to hold. Consequently, in the decomposition of the solution, one has two other terms that decay more slowly with rates $t^{-1 / \alpha}$ and $\max \left\{t^{-r_{j} / \alpha_{j}} ; 1 \leqslant j \leqslant J\right\}$. Note also that, necessarily, $r^{j} / \alpha_{j}<m / 2$ because $r_{j}<m$ and $\alpha \geqslant 2$ whenever $\mathcal{D}\left(A_{1}, \ldots, A_{m}, B\right)$ is not trivial.

Proof of Proposition 7 : In order to simplify the notations in this proof, we write $\mathcal{D}$ instead of $\mathcal{D}\left(A_{1}, \ldots, A_{m}, B\right)$.

Let $d \in(0,1)$. We define $w_{1}, w_{2}, w_{3}, w_{4}$ by the following expressions, for every $t \in(0,+\infty)$ and for every $\xi \in \mathbb{R}^{*}$,

$$
\begin{aligned}
& \hat{w}_{1}(t, \xi):=\hat{w}(t, \xi) 1_{\rho>1} 1_{\operatorname{dist}(\omega, \mathcal{D})>d} \\
& \hat{w}_{2}(t, \xi):=\hat{w}(t, \xi) 1_{\rho<1} 1_{\operatorname{dist}(\omega, \mathcal{D})>d} \\
& w_{3}:=\sum_{j=1}^{J} w_{3, j} \\
& \hat{w}_{3, j}(t, \xi):=\hat{w}(t, \xi) 1_{\rho>1} 1_{\operatorname{dist}(\omega, \mathcal{D})=\operatorname{dist}\left(\omega, \mathcal{D}_{j}\right)<d} \\
& w_{4}:=\sum_{j=1}^{J} w_{4, j} \\
& \hat{w}_{4, j}(t, \xi):=\hat{w}(t, \xi) 1_{\rho<1} 1_{\operatorname{dist}(\omega, \mathcal{D})=\operatorname{dist}\left(\omega, \mathcal{D}_{j}\right)<d} .
\end{aligned}
$$

Thanks to Proposition 1 and assumption (A2), there exists $c>0$ depending on $d, c_{j}, \alpha_{j}$ such that, for every $\xi \in \mathbb{R}^{m}$,

$$
\begin{aligned}
& \left|\hat{w}_{1}(t, \xi)\right| \leqslant \sqrt{3}\left|\hat{w}^{0}(\xi)\right| e^{-c t} \\
& \left|\hat{w}_{2}(t, \xi)\right| \leqslant \sqrt{3}\left|\hat{w}^{0}(\xi)\right| e^{-c \rho^{2} t} .
\end{aligned}
$$

The same computations as in the proof of Theorem 1 lead to

$$
\begin{aligned}
& \left\|w_{1}(t)\right\|_{L^{2}\left(\mathbb{R}^{m}, \mathbb{R}^{n}\right)} \leqslant \sqrt{3}\left\|w^{0}\right\|_{L^{2}\left(\mathbb{R}^{m}, \mathbb{R}^{n}\right)} e^{-c t}, \\
& \left\|w_{2}(t)\right\|_{L^{\infty}\left(\mathbb{R}^{m}, \mathbb{R}^{n}\right)} \leqslant \frac{C}{t^{m / 2}}\left\|w^{0}\right\|_{L^{1}\left(\mathbb{R}^{m}, \mathbb{R}^{n}\right)} .
\end{aligned}
$$

We fix $j \in\{1, \ldots, J\}$. In the study of $w_{3, j}$ and $w_{4, j}$, we will use new coordinates on $\mathbb{R}^{m}$. We introduce the orthogonal sum

$$
\mathbb{R}^{m}=\mathcal{D}_{j}^{\perp}+\mathcal{D}_{j}: \xi=\xi^{\prime}+\xi^{\prime \prime}
$$


We fix an orthonormal basis $\left(b_{1}^{\prime}, \ldots, b_{r_{j}}^{\prime}\right)$ of $\mathcal{D}_{j}^{\perp}$ and another orthonormal basis $\left(b_{1}^{\prime \prime}, \ldots, b_{m-r_{j}}^{\prime \prime}\right)$ of $\mathcal{D}_{j}$. For $\xi=\left(\xi_{1}, \ldots, \xi_{m}\right)^{t} \in \mathbb{R}^{m}-\{0\}$, let $\rho:=|\xi| \in \mathbb{R}_{+}^{*}$, $\theta \in[0, \pi / 2]$ be such that

$$
\left|\xi^{\prime}\right|=\rho \cos (\theta) \text { i.e. } \theta:=\arccos \left(\frac{\left|\xi^{\prime}\right|}{|\xi|}\right) .
$$

Then $\xi^{\prime}=\rho \cos (\theta) \zeta^{*}, \xi^{\prime \prime}=\rho \sin (\theta) \zeta^{* *}$, where $\zeta^{*} \in \mathcal{D}_{j}^{\perp} \cap S^{m-1}, \zeta^{* *} \in \mathcal{D}_{j} \cap$ $S^{m-1}$. There exists a unique pair $(\alpha, \beta)$ with $\alpha=\left(\alpha_{1}, \alpha_{2}, \ldots, \alpha_{r_{j}-1}\right) \in \Omega_{\alpha}:=$ $[-\pi / 2, \pi / 2) \times[0,2 \pi)^{r_{j}-2}, \beta=\left(\beta_{1}, \beta_{2}, \ldots, \beta_{m-r_{j}-1}\right) \in \Omega_{\beta}:=[-\pi / 2, \pi / 2) \times$ $[0,2 \pi)^{m-r_{j}-2}$, such that

$$
\begin{aligned}
\zeta^{*}= & \cos \left(\alpha_{1}\right) \cos \left(\alpha_{2}\right) \ldots \cos \left(\alpha_{r_{j}-2}\right) \cos \left(\alpha_{r_{j}-1}\right) b_{1}^{\prime}+ \\
& \cos \left(\alpha_{1}\right) \cos \left(\alpha_{2}\right) \ldots \cos \left(\alpha_{r_{j}-2}\right) \sin \left(\alpha_{r_{j}-1}\right) b_{2}^{\prime}+ \\
& \cos \left(\alpha_{1}\right) \cos \left(\alpha_{2}\right) \ldots \sin \left(\alpha_{r_{j}-2}\right) b_{3}^{\prime}+ \\
& \ldots \\
& \ldots \\
& \quad \cos \left(\alpha_{1}\right) \sin \left(\alpha_{2}\right) b_{r_{j}-1}^{\prime}+ \\
& \quad \sin \left(\alpha_{1}\right) b_{r_{j}}^{\prime}, \\
\zeta^{* *}= & \cos \left(\alpha_{1}\right) \cos \left(\alpha_{2}\right) \ldots \cos \left(\alpha_{m-r_{j}-2}\right) \cos \left(\alpha_{m-r_{j}-1}\right) b_{1}^{\prime \prime}+ \\
& \cos \left(\alpha_{1}\right) \cos \left(\alpha_{2}\right) \ldots \cos \left(\alpha_{m-r_{j}-2}\right) \sin \left(\alpha_{m-r_{j}-1}\right) b_{2}^{\prime \prime}+ \\
& \cos \left(\alpha_{1}\right) \cos \left(\alpha_{2}\right) \ldots \sin \left(\alpha_{m-r_{j}-2}\right) b_{3}^{\prime \prime}+ \\
& \ldots \\
& \ldots \\
& \cos \left(\alpha_{1}\right) \sin \left(\alpha_{2}\right) b_{m-r_{j}-1}^{\prime \prime}+ \\
& \sin \left(\alpha_{1}\right) b_{m-r_{j}}^{\prime \prime} .
\end{aligned}
$$

In the following arguments, we will use the change of variables $\left(\xi_{1}, \ldots, \xi_{m}\right) \rightarrow$ $(\rho, \theta, \alpha, \beta),\left(\xi_{1}, \ldots, \xi_{m}\right) \rightarrow\left(\xi^{\prime}, \xi^{\prime \prime}\right)$. We have

$$
d \xi_{1} \ldots d \xi_{m}=d \xi^{\prime} d \xi^{\prime \prime}
$$

because $\xi=\left(\xi_{1}, \ldots, \xi_{m}\right) \mapsto\left(\xi^{\prime}, \xi^{\prime \prime}\right)$ is a unitary linear transformation. We have

$$
d \xi_{1} \ldots d \xi_{m}=J(\rho, \theta, \alpha, \beta) d \rho d \theta d \alpha d \beta
$$

where $J$ is the Jacobian of the change of variables. Since the dependence of $\xi$ with respect to $\theta, \alpha_{1}, \ldots, \alpha_{r_{j}-1}, \beta_{1}, \ldots, \beta_{m-r_{j}-1}$ always involves the functions $\cos ($.$) and \sin ($.$) , there exists C=C\left(\mathcal{D}_{j}\right)>0$ such that,

$$
|J(\rho, \theta, \alpha, \beta)| \leqslant C \rho^{m-1} d \rho d \theta d \alpha d \beta .
$$

Now, let us compute a bound on $w_{3, j}$. For $\xi \in \mathbb{R}^{m}$, we have the orthogonal decomposition

$$
\begin{aligned}
\mathbb{R}^{m} & =\mathcal{D}_{j}^{\perp}+\mathcal{D}_{j} \\
\frac{\xi}{|\xi|} & =\frac{\xi^{\prime}}{|\xi|}+\frac{\xi^{\prime \prime}}{|\xi|}
\end{aligned}
$$

thus

$$
\frac{\left|\xi^{\prime}\right|}{|\xi|}=\operatorname{dist}\left(\frac{\xi}{|\xi|}, \mathcal{D}_{j}\right)
$$


therefore

$$
\begin{aligned}
D_{3, j} & :=\left\{\xi \in \mathbb{R}^{m} ;|\xi|>1, \operatorname{dist}\left(\frac{\xi}{|\xi|} ; \mathcal{D}\right)=\operatorname{dist}\left(\frac{\xi}{|\xi|} ; \mathcal{D}_{j}\right)<d\right\} \\
& =\left\{\xi \in \mathbb{R}^{m} ;|\xi|>1, \frac{\left|\xi^{\prime}\right|}{|\xi|}<d\right\} \\
& =\left\{\xi \in \mathbb{R}^{m} ; \rho>1, \cos (\theta)<d\right\} .
\end{aligned}
$$

Using the previous inclusion, Proposition 1, the assumption (48) and finally (53), we get

$$
\begin{aligned}
\left\|w_{3, j}(t)\right\|_{L^{\infty}} & \leqslant\left\|\hat{w}_{3, j}(t)\right\|_{L^{1}} \\
& \leqslant \int_{D_{3, j}}|\hat{w}(t, \xi)| d \xi \\
& \leqslant \int_{D_{3, j}} \sqrt{3}\left|\hat{w}^{0}(\xi)\right| e^{-c N_{*}(\xi /|\xi|) t} d \xi \\
& \leqslant \int_{D_{3, j}} \sqrt{3}\left|\hat{w}^{0}(\xi)\right| e^{-c c_{j} \operatorname{dist}\left(\xi /|\xi|, \mathcal{D}_{j}\right)^{\alpha_{j}}} d \xi \\
& \leqslant \int_{D_{3, j}} \sqrt{3}\left|\hat{w}^{0}(\xi)\right| e^{-c c_{j}\left(\left|\xi^{\prime}\right| /|\xi|\right)^{\alpha_{j}} t} d \xi .
\end{aligned}
$$

Now, we perform a change of variable $\left(\xi_{1}, \ldots, \xi_{m}\right) \rightarrow(\rho, \theta, \alpha, \beta)$. Thanks to (52), we have

$$
\left\|w_{3, j}(t)\right\|_{L^{\infty}} \leqslant \int_{1}^{\infty} \int_{\theta(d)}^{\pi / 2} \int_{\Omega_{\alpha}} \int_{\Omega_{\beta}} C \sqrt{3} L\left[\hat{w}^{0}\right](\rho) e^{-c c_{j} \cos (\theta)^{\alpha_{j}}} \rho^{m-1} d \rho d \theta d \alpha d \beta,
$$

where $\theta(d):=\arccos (d)$ (indeed $\left|\xi^{\prime}\right| /|\xi|=\cos (\theta)<d$ in $\left.D_{3, j}\right)$. Therefore, we have

$$
\left\|w_{3, j}(t)\right\|_{L^{\infty}} \leqslant C_{1} N_{1}\left(w^{0}\right) \int_{\theta(d)}^{\pi / 2} e^{-c c_{j} \cos (\theta)^{\alpha_{j}} t} d \theta
$$

where $C_{1}:=C \sqrt{3} \operatorname{vol}\left(\Omega_{\alpha}\right) \operatorname{vol}\left(\Omega_{\beta}\right)$. Performing the change of variable

$$
y=\cos (\theta) t^{1 / \alpha_{j}} \quad \text { i.e. } \quad \theta=\arccos \left(\frac{y}{t^{1 / \alpha_{j}}}\right),
$$

we get

$$
\begin{aligned}
\int_{\theta(d)}^{\pi / 2} e^{-c c_{j} \cos (\theta)^{\alpha_{j}} t} d \theta & =\int_{0}^{d t^{1 / \alpha_{j}}} e^{-c c_{j} y^{\alpha_{j}}} \frac{1}{\sqrt{1-\frac{y^{2}}{t^{2 / \alpha_{j}}}}} \frac{d y}{t^{1 / \alpha_{j}}} \\
& \leqslant \frac{1}{t^{1 / \alpha_{j}}} \frac{1}{\sqrt{1-d^{2}}} \int_{0}^{\infty} e^{-c c_{j} y^{\alpha_{j}}} d y .
\end{aligned}
$$

This proves (50) with any constant $C$ such that

$$
C \geqslant \frac{C_{1}}{\sqrt{1-d^{2}}} \sum_{j=1}^{J} \int_{0}^{\infty} e^{-c c_{j} y^{\alpha_{j}}} d y .
$$

Now, let us compute a bound on $w_{4, j}$. With the same strategy, we get

$$
\begin{aligned}
\left\|w_{4, j}(t)\right\|_{L^{\infty}} & \leqslant \int_{D_{4, j}} \sqrt{3}\left|\hat{w}^{0}(\xi)\right| e^{-c|\xi|^{2} N_{*}(\xi /|\xi|) t} d \xi \\
& \leqslant \int_{D_{4, j}} \sqrt{3}\left|\hat{w}^{0}(\xi)\right| e^{-c c_{j}|\xi|^{2}\left(\left|\xi^{\prime}\right| /|\xi|\right)^{\alpha_{j}}} d \xi
\end{aligned}
$$

where

$$
D_{4, j}:=\left\{\xi \in \mathbb{R}^{m} ;|\xi|<1, \operatorname{dist}(\xi /|\xi|, \mathcal{D})=\operatorname{dist}\left(\xi /|\xi|, \mathcal{D}_{j}\right)<d\right\} .
$$


Using $\left\|\hat{w}^{0}\right\|_{L^{\infty}} \leqslant\left\|w^{0}\right\|_{L^{1}}$, the inequality $|\xi|^{2} \cos (\theta)^{\alpha_{j}} \geqslant[|\xi| \cos (\theta)]^{\alpha_{j}}=\left|\xi^{\prime}\right|^{\alpha_{j}}$ (because $\alpha_{j} \geqslant 2$ ) and the inclusion

$$
D_{4, j} \subset\left\{\xi \in \mathbb{R}^{m} ;\left|\xi^{\prime}\right|<d,\left|\xi^{\prime \prime}\right|<1\right\},
$$

we get

$$
\left\|w_{4, j}(t)\right\|_{L^{\infty}} \leqslant \sqrt{3}\left\|w^{0}\right\|_{L^{1}} \operatorname{vol}\left(B_{\mathbb{R}^{m-r_{j}}}(0,1)\right) \int_{\xi^{\prime} \in B_{\mathbb{R}^{r_{j}}}(0, d)} e^{-c c_{j}\left|\xi^{\prime}\right|^{\alpha_{j}} t} d \xi^{\prime} .
$$

Performing the change of variable $\zeta=t^{1 / \alpha_{j}} \xi^{\prime}$, we get

$$
\int_{\xi^{\prime} \in B_{\mathbb{R}^{r}}(0, d)} e^{-c \tilde{c}\left|\xi^{\prime}\right|^{\alpha_{j}} t} d \xi^{\prime} \leqslant \frac{1}{t^{r_{j} / \alpha_{j}}} \int_{\mathbb{R}^{r}} e^{-c c_{j}|\zeta|^{\alpha_{j}}} d \zeta,
$$

which gives (51) with any constant $C$ such that

$$
C \geqslant \sqrt{3} \sum_{j=1}^{J} \operatorname{vol}\left(B_{\mathbb{R}^{m-r_{j}}}(0,1)\right) \int_{\mathbb{R}^{r}} e^{-c c_{j}|\zeta|^{\alpha_{j}}} d \zeta .
$$

Remark 14 Extending the validity of this kind of decomposition to more general situations (i.e. when the algebraic submanifold $\mathcal{D}\left(A_{1}, \ldots, A_{m}, B\right)$ is not the union of a finite number of vector subspaces) is an open problem. Indeed, the strategy used in the proof would need a parameterization of the submanifold $\mathcal{D}\left(A_{1}, \ldots, A_{m}, B\right)$. In this case the conditions to be imposed on the initial datum to ensure a similar decomposition are also to be clarified.

\subsection{Decomposition when $n_{1}=1$}

This subsection is devoted to study the function $\omega \in S^{m-1} \mapsto N_{*, \epsilon}(\omega)$ for $\epsilon>0$, defined in Proposition 1 in the particular case $n_{1}=1$ (i.e. $\operatorname{Ker}(B)=\operatorname{Span}\left(e_{1}\right)$ ). The parameters $\epsilon, m_{0}, \ldots, m_{n-1}$ are fixed as in Proposition 1.

Proposition 8 Let $A_{1}, \ldots, A_{m}, B$ be $n * n$ real matrices with $A_{1}, \ldots, A_{m}$ symmetric and $B$ of the form (5) with $n_{1}=1$. Then

- (1) $\mathcal{D}\left(A_{1}, \ldots, A_{m}, B\right)$ is a vector subspace,

- (2) there exists $\epsilon_{*}=\epsilon_{*}\left(A_{1}, \ldots, A_{m}, B\right)>0$ such that, for every $\epsilon \in\left(0, \epsilon_{*}\right)$, there exists $c=c\left(\epsilon, A_{1}, \ldots, A_{m}, B\right)$ such that, for every $\omega \in S^{m-1}$,

$$
N_{*, \epsilon}(\omega) \geqslant \operatorname{cdist}(\omega, \mathcal{D})^{2} .
$$

A direct consequence of Proposition 8 and Proposition 7 is the following theorem.

Theorem 2 Under the same assumptions as in Proposition 8, there exists $C, \lambda>0$ such that, for every $w^{0} \in L^{1} \cap L^{2}\left(\mathbb{R}^{m}, \mathbb{R}^{n}\right)$ with (49), the solution of (4) with initial condition $w^{0}$ can be decomposed as

$$
w=w_{1}+w_{2}+w_{3}+w_{4}
$$


where (14) holds and

$$
\begin{gathered}
\left\|w_{3}(t)\right\|_{L^{\infty}\left(\mathbb{R}^{m}, \mathbb{R}^{n}\right)} \leqslant \frac{C}{\sqrt{t}} N_{1}\left(w^{0}\right), \forall t \in(0,+\infty) \\
\left.\left\|w_{4}(t)\right\|_{L^{\infty}\left(\mathbb{R}^{m}, \mathbb{R}^{n}\right)} \leqslant \frac{C}{t^{\frac{r}{2}}}\left\|w^{0}\right\|_{L^{1}\left(\mathbb{R}^{m}, \mathbb{R}^{n}\right)}\right), \forall t \in(0,+\infty),
\end{gathered}
$$

where $r:=\operatorname{codim}\left(\mathcal{D}\left(A_{1}, \ldots, A_{m}, B\right)\right.$.

Proof of Proposition 8: The assumption $n_{1}=1$ implies that

$$
\begin{aligned}
\mathcal{D}\left(A_{1}, \ldots, A_{m}, B\right) & =\left\{\xi \in \mathbb{R}^{m} ; e_{1} \text { is an eigenvector of } A(\xi)\right\} \\
& =\left\{\xi \in \mathbb{R}^{m} ; \sum_{j=1}^{m} a_{k, 1}^{(j)} \xi_{j}=0, \forall k=2, \ldots, n\right\},
\end{aligned}
$$

which proves (1).

Let $\epsilon_{*}>0$ be such that

$$
\begin{gathered}
\frac{2 \mathcal{M}_{1}^{2}}{c} \epsilon_{*}^{m_{1}}<\frac{1}{2}, \\
\epsilon_{*}^{m_{1}} \sum_{k=2}^{n}\left(\sum_{j=1}^{m}\left|a_{k, 1}^{(j)}\right|\right)^{2}<1,
\end{gathered}
$$

where $\mathcal{M}_{1}$ is defined by $(27)$ and $c=c(B)$ is such that $|B v|^{2} \geqslant c|v|^{2}, \forall v \in$ $\operatorname{Span}\left(e_{2}, \ldots, e_{n}\right)$. We have

$$
\begin{aligned}
N_{*}(\omega) & =\min \left\{\sum_{k=0}^{n-1} \epsilon^{m_{k}}\left|B A(\omega)^{k} x\right|^{2} ; x \in S^{n-1}\right\} \\
& \geqslant \min \left\{|B x|^{2}+\epsilon^{m_{1}}|B A(\omega) x|^{2} ; x \in S^{n-1}\right\} .
\end{aligned}
$$

Let $\omega \in \mathbb{R}^{m}-\mathcal{D}\left(A_{1}, \ldots, A_{m}, B\right)$ and $x \in S^{n-1}$. We consider the decomposition $x=\beta e_{1}+x_{\perp}$ where $\beta \in \mathbb{R}$ and $x_{\perp} \in \operatorname{Span}\left(e_{2}, \ldots, e_{n}\right)$. Thanks to the definition of $c$ and (27), we have

$$
\begin{aligned}
& |B x|^{2}+\epsilon^{m_{1}}|B A(\omega) x|^{2} \\
= & \left|B x_{\perp}\right|^{2}+\beta^{2} \epsilon^{m_{1}}\left|B A(\omega) e_{1}\right|^{2}+\epsilon^{m_{1}}\left|B A(\omega) x_{\perp}\right|^{2}+2 \beta \epsilon^{m_{1}}\left\langle B A(\omega) e_{1}, B A(\omega) x_{\perp}\right\rangle \\
\geqslant & c\left|x_{\perp}\right|^{2}+\beta^{2} \epsilon^{m_{1}}\left|B A(\omega) e_{1}\right|^{2}-2|\beta| \epsilon^{m_{1}}\left|B A(\omega) e_{1}\right| \mathcal{M}_{1}\left|x_{\perp}\right| \\
\geqslant & \frac{c}{2}\left|x_{\perp}\right|^{2}+\beta^{2} \epsilon^{m_{1}}\left(1-\frac{2 \mathcal{M}_{1}^{2}}{c} \epsilon^{m_{1}}\right)\left|B A(\omega) e_{1}\right|^{2} \\
\geqslant & \frac{c}{2}\left|x_{\perp}\right|^{2}+\frac{\beta^{2}}{2} \epsilon^{m_{1}}\left|B A(\omega) e_{1}\right|^{2} .
\end{aligned}
$$

Since $B e_{1}=0$, we have

$$
B A(\omega) e_{1}=B\left(\begin{array}{c}
0 \\
\sum_{j=1}^{m} a_{2,1}^{(j)} \omega_{j} \\
\cdots \\
\sum_{j=1}^{m} a_{n, 1}^{(j)} \omega_{j}
\end{array}\right)
$$

thus there exists a positive constant $c=c(B)$ such that

$$
\left|B A(\omega) e_{1}\right|^{2} \geqslant c \sum_{k=2}^{n}\left|\sum_{j=1}^{m} a_{k, 1}^{(j)} \omega_{j}\right|^{2} .
$$


Therefore,

$$
|B x|^{2}+\epsilon^{m_{1}}|B A(\omega) x|^{2} \geqslant \frac{c}{2} \min \left\{1 ; \epsilon^{m_{1}} \sum_{k=2}^{n}\left|\sum_{j=1}^{m} a_{k, 1}^{(j)} \omega_{j}\right|^{2}\right\}\left(\left|x_{\perp}\right|^{2}+\beta^{2}\right),
$$

thus

$$
N_{*}(\omega) \geqslant \frac{\epsilon^{m_{1}} c}{2} \sum_{k=2}^{n}\left|\sum_{j=1}^{m} a_{k, 1}^{(j)} \omega_{j}\right|^{2} .
$$

In order to conclude, let us prove the existence of a constant $C>0$ such that

$$
\sum_{k=2}^{n}\left|\sum_{j=1}^{m} a_{k, 1}^{(j)} \omega_{j}\right|^{2} \geqslant C \operatorname{dist}(\omega, \mathcal{D})^{2} .
$$

Let $\mathcal{D}^{\perp}$ be the orthogonal supplementary of the subspace $\mathcal{D}$. Thanks to (56), the quadratic form

$$
\tilde{\omega} \in \mathcal{D}^{\perp} \mapsto \sum_{k=2}^{n}\left|\sum_{j=1}^{m} a_{k, 1}^{(j)} \tilde{\omega}_{j}\right|^{2}
$$

is positive definite on $\mathcal{D}^{\perp}$. Thus, there exists $C>0$ such that

$$
\sum_{k=2}^{n}\left|\sum_{j=1}^{m} a_{k, 1}^{(j)} \tilde{\omega}_{j}\right|^{2} \geqslant C|\tilde{\omega}|^{2}, \forall \tilde{\omega} \in \mathcal{D}^{\perp}
$$

Using the decomposition

$$
\begin{aligned}
& \mathbb{R}^{m}=\mathcal{D} \oplus \mathcal{D}^{\perp} \\
& \omega=\omega^{\sharp}+\tilde{\omega}
\end{aligned}
$$

we get

$$
\sum_{k=2}^{n}\left|\sum_{j=1}^{m} a_{k, 1}^{(j)} \omega_{j}\right|^{2}=\sum_{k=2}^{n}\left|\sum_{j=1}^{m} a_{k, 1}^{(j)} \tilde{\omega}_{j}\right|^{2} \geqslant C|\tilde{\omega}|^{2}=C \operatorname{dist}(\omega, \mathcal{D})^{2}, \forall \omega \in S^{m-1} . \square
$$

\subsection{Discussion on explicit examples}

This section is devoted to the study of explicit examples, and discussing the optimal value of the exponents $\alpha_{j}$ in the assumption (48) of Proposition 7 . Note that the optimal values of the exponents $\alpha_{j}$ are important in the study of the asymptotic behavior of (4) because they appear in the decomposition of these solutions (see Proposition 7).

Consider the case $m \geqslant 2$,

$$
A_{1}:=\left(\begin{array}{ccc}
0 & 1 & 0 \\
1 & 0 & 0 \\
0 & 0 & 0
\end{array}\right), \quad A_{2}:=\left(\begin{array}{ccc}
0 & 0 & 1 \\
0 & 0 & 0 \\
1 & 0 & 0
\end{array}\right), \quad B:=\left(\begin{array}{ccc}
0 & 0 & 0 \\
0 & 0 & 0 \\
0 & 0 & 1
\end{array}\right),
$$

$A_{k}=0$ for $k=3, \ldots, m$ and the associated system (4) with $\xi \in \mathbb{R}^{m}$. Then, we have $n_{1}=2$ and, for every $\xi=\left(\xi_{1}, \ldots, \xi_{m}\right)^{t} \in \mathbb{R}^{m}$,

$$
A(\xi)=\sum_{j=1}^{m} \xi_{j} A_{j}=\left(\begin{array}{ccc}
0 & \xi_{1} & \xi_{2} \\
\xi_{1} & 0 & 0 \\
\xi_{2} & 0 & 0
\end{array}\right) .
$$


Thus

$$
\mathcal{D}=\mathcal{D}_{1} \cup \mathcal{D}_{2}
$$

where

$$
\mathcal{D}_{1}:=\left\{\xi \in \mathbb{R}^{m} ; \xi_{1}=0\right\}, \mathcal{D}_{2}:=\left\{\xi \in \mathbb{R}^{m} ; \xi_{2}=0\right\} .
$$

(see the analysis made in Example 2.a in Section 3.2).

Proposition 9 There exists $\epsilon_{*}>0$ such that, for every $\epsilon \in\left(0, \epsilon_{*}\right)$,

(1) with $m=2$, there exists $c>0$ such that,

$$
N_{*}(\omega) \geqslant \operatorname{cdist}(\omega, \mathcal{D})^{2}, \forall \omega \in S^{1} .
$$

(2) with $m \geqslant 3$, (57) fails but there exists $c>0$ such that

$$
N_{*}(\omega) \geqslant \operatorname{cdist}(\omega, \mathcal{D})^{4}, \forall \omega \in S^{m-1} \text {. }
$$

The previous proposition leads to the following remarks

- The assumption $n_{1}=1$ is not necessary for (57) to hold. Indeed, it holds for the example studied in this section with $m=2$ and in that case $n_{1}=2$.

- The power $\alpha_{j}$ in (48), that coincides with 2 when $n_{1}=1$, may be strictly greater than 2 when $n_{1} \geqslant 2$. Indeed, for the example studied in this section with $m \geqslant 3$, (48) holds with $\alpha_{j}=4$ but not with $\alpha_{j}=2$.

Propositions 9 and 7 lead to the following decomposition for the solutions of (4) with the previous matrices.

Theorem 3 Let $m \geqslant 2$ and $A_{1}, \ldots, A_{m}, B$ be defined at the beginning of this section. There exists $C, \lambda>0$ such that, for every $w^{0} \in L^{1} \cap L^{2}\left(\mathbb{R}^{m}, \mathbb{R}^{n}\right)$ with (49), the solution of (4) with initial condition $w^{0}$ can be decomposed as

$$
w=w_{1}+w_{2}+w_{3}+w_{4}
$$

where (14) holds and

- when $m=2$,

$$
\begin{gathered}
\left\|w_{3}(t)\right\|_{L^{\infty}\left(\mathbb{R}^{m}, \mathbb{R}^{n}\right)} \leqslant \frac{C}{\sqrt{t}} N_{1}\left(w^{0}\right), \forall t \in(0,+\infty), \\
\left.\left\|w_{4}(t)\right\|_{L^{\infty}\left(\mathbb{R}^{m}, \mathbb{R}^{n}\right)} \leqslant \frac{C}{t^{\frac{m-1}{2}}}\left\|w^{0}\right\|_{L^{1}\left(\mathbb{R}^{m}, \mathbb{R}^{n}\right)}\right), \forall t \in(0,+\infty),
\end{gathered}
$$

- when $m \geqslant 3$,

$$
\begin{gathered}
\left\|w_{3}(t)\right\|_{L^{\infty}\left(\mathbb{R}^{m}, \mathbb{R}^{n}\right)} \leqslant \frac{C}{t^{\frac{1}{4}}} N_{1}\left(w^{0}\right), \forall t \in(0,+\infty), \\
\left.\left\|w_{4}(t)\right\|_{L^{\infty}\left(\mathbb{R}^{m}, \mathbb{R}^{n}\right)} \leqslant \frac{C}{t^{\frac{m-1}{4}}}\left\|w^{0}\right\|_{L^{1}\left(\mathbb{R}^{m}, \mathbb{R}^{n}\right)}\right), \forall t \in(0,+\infty) .
\end{gathered}
$$


Proof of Proposition 9 : Let $\epsilon_{*}>0$ be such that

$$
\epsilon_{*}^{m_{2}}<\frac{1}{2}
$$

and $\epsilon \in\left(0, \epsilon_{*}\right)$. Easy computations lead to

$$
M_{\epsilon}(\omega)=\sum_{k=0}^{2} \epsilon^{m_{k}} A(\omega)^{k} B^{t} B A(\omega)^{k}=\left(\begin{array}{ccc}
\epsilon^{m_{1}} \omega_{2}^{2} & 0 & 0 \\
0 & \epsilon^{m_{2}} \omega_{1}^{2} \omega_{2}^{2} & \epsilon^{m_{2}} \omega_{1} \omega_{2}^{3} \\
0 & \epsilon^{m_{2}} \omega_{1} \omega_{2}^{3} & 1+\epsilon^{m_{2}} \omega_{2}^{4}
\end{array}\right)
$$

Thus, for every $v \in S^{2}$, we have

$$
\begin{aligned}
v^{t} M(\omega) v & =\epsilon^{m_{1}} \omega_{2}^{2} v_{1}^{2}+\epsilon^{m_{2}} \omega_{1}^{2} \omega_{2}^{2} v_{2}^{2}+2 \epsilon^{m_{2}} \omega_{1} \omega_{2}^{3} v_{2} v_{3}+\left[1+\epsilon^{m_{2}} \omega_{2}^{4}\right] v_{3}^{2} \\
& \geqslant \epsilon^{m_{1}} \omega_{2}^{2} v_{1}^{2}+\frac{1}{2} \epsilon^{m_{2}} \omega_{1}^{2} \omega_{2}^{2} v_{2}^{2}+\left[1-\epsilon^{m_{2}} \omega_{2}^{4}\right] v_{3}^{2} \\
& \geqslant \min \left\{\epsilon^{m_{1}} \omega_{2}^{2}, \frac{1}{2} \epsilon^{m_{2}} \omega_{1}^{2} \omega_{2}^{2}, 1-\epsilon^{m_{2}} \omega_{2}^{4}\right\}
\end{aligned}
$$

Noticing that, for every $\omega \in S^{m-1}$,

$$
1-\epsilon^{m_{2}} \omega_{2}^{4} \geqslant 1-\epsilon^{m_{2}}>\frac{1}{2}>\frac{1}{2} \epsilon^{m_{2}} \omega_{1}^{2} \omega_{2}^{2},
$$

and using the definition (24), we get

$$
N_{*}(\omega) \geqslant \min \left\{\epsilon^{m_{1}} \omega_{2}^{2}, \frac{1}{2} \epsilon^{m_{2}} \omega_{1}^{2} \omega_{2}^{2}\right\} .
$$

1st case : $m=2$. For every $\omega \in S^{1}$, we have $\omega_{1}^{2}+\omega_{2}^{2}=1$ thus (64) leads to

$$
N_{*}(\omega) \geqslant \min \left\{\epsilon^{m_{1}}, \frac{1}{4} \epsilon^{m_{2}}\right\} \operatorname{dist}(\omega, \mathcal{D})^{2} .
$$

2nd case : $m \geqslant 3$. Let $\omega \in S^{m-1}$ and $j \in\{1,2\}$ be such that $\operatorname{dist}(\omega, \mathcal{D})=$ $\operatorname{dist}\left(\omega, \mathcal{D}_{j}\right)$. Then $\left|\omega_{j}\right| \leqslant\left|\omega_{i}\right| \leqslant 1$ for $i \in\{1,2\}$ such that $i \neq j$, thus (64) leads to

$$
N_{*}(\omega) \geqslant \min \left\{\epsilon^{m_{1}}, \frac{1}{2} \epsilon^{m_{2}}\right\}\left|\omega_{j}\right|^{4}=\min \left\{\epsilon^{m_{1}}, \frac{1}{2} \epsilon^{m_{2}}\right\} \operatorname{dist}(\omega, \mathcal{D})^{4} .
$$

Now, let us show that (57) may not hold with $m \geqslant 3$. We consider $\omega \in S^{m-1}$ of the form

$$
\omega=\left(\begin{array}{c}
\omega_{1} \\
2 \omega_{1} \\
\sqrt{1-5 \omega_{1}^{2}} \\
0 \\
\cdots \\
0
\end{array}\right),
$$

where $\omega_{1}>0$ is small. Using the definition of $N_{*, \epsilon}(\omega)$ given by $(24)$ and the explicit expression of the matrix $M_{\epsilon}(\omega)$ given by $(63)$, we get

$$
N_{*}(\omega) \leqslant e_{2}^{t} M_{\epsilon}(\omega) e_{2}=4 \epsilon^{m_{2}} \omega_{1}^{4}=4 \epsilon^{m_{2}} \operatorname{dist}(\omega, \mathcal{D})^{4} .
$$

We conclude by considering points $\omega \in S^{m-1}$ of the form (65), with $\omega_{1} \rightarrow 0$. 


\subsection{Complete classification in the case $n=2$}

Let us consider the system (4) with

$$
A_{j}:=\left(\begin{array}{ll}
a_{1}^{(j)} & a_{1,2}^{(j)} \\
a_{1,2}^{(j)} & a_{2}^{(j)}
\end{array}\right), \quad B:=\left(\begin{array}{cc}
0 & 0 \\
0 & 1
\end{array}\right),
$$

with $a_{1}^{(j)}, a_{1,2}^{(j)}, a_{2,1}^{(j)}, a_{2}^{(j)} \in \mathbb{R}$. All possible partially dissipated systems with $n=2$ enter in this setting. Obviously, in this case, $n_{1}=n_{2}=1$.

Theorem 4 Let $m \in \mathbb{N}^{*}, m \geqslant 2$, and $A_{1}, \ldots, A_{m}, B$ be real matrices of the form (66).

(A) When $a_{1,2}^{(j)}=0$, for $j=1, \ldots, m$, then, $\mathcal{D}\left(A_{1}, \ldots, A_{m}, B\right)=\mathbb{R}^{m}$, and, for every solution of (4) with $L^{2}\left(\mathbb{R}^{m}, \mathbb{R}^{2}\right)$ initial condition, the first component is a traveling wave and the second one decays exponentially in $L^{2}$.

(B) Let us assume that there exists $j \in\{1, \ldots, m\}$ such that $a_{1,2}^{(j)} \neq 0$. Then $\mathcal{D}\left(A_{1}, \ldots, A_{m}, B\right)=\left\{\xi \in \mathbb{R}^{m} ; \sum_{j=0}^{m} a_{1,2}^{(j)} \xi_{j}=0\right\}$ is an hyperplane of $\mathbb{R}^{m}$ and there exist $C=C\left(A_{1}, \ldots, A_{m}, B\right)>0$ and $\lambda=\lambda\left(A_{1}, \ldots, A_{m}, B\right)>0$ such that, for every $w^{0} \in L^{1} \cap L^{2}\left(\mathbb{R}^{m}, \mathbb{R}^{2}\right)$ with (49), the solution of (4) with initial condition $w^{0}$ can be decomposed as

$$
w=w_{1}+w_{2}+w_{3}
$$

where (14) holds and

$$
\left\|w_{3}(t)\right\|_{L^{\infty}\left(\mathbb{R}^{m}, \mathbb{R}^{2}\right)} \leqslant \frac{C}{\sqrt{t}}\left(N_{1}\left(w^{0}\right)+\left\|w^{0}\right\|_{L^{1}\left(\mathbb{R}^{m}, \mathbb{R}^{n}\right)}\right)
$$

Proof of Proposition 4 : (A) can be obtained from easy explicit computations and $(\mathbf{B})$ is a consequence of Theorem 2 .

\section{Summary array of the classification, open prob- lems and conjectures}

\subsection{Summary array of the classification}

We recapitulate the results proved in Sections 2, 3 and 4 in the following array. 


\begin{tabular}{|c|c|c|c|l|}
\hline$m, n$ & $(S K)$ & $\mathcal{D}$ & $L^{2}$ stability & decomposition \\
\hline$m=1$ & yes & $\{0\}$ & yes & $e^{-t}+\frac{1}{\sqrt{t}}$ \\
$\forall n$ & no & $\mathbb{R}$ & no & $e^{-t}+\frac{1}{\sqrt{t}}+$ tr. wave \\
\hline$n=2$ & yes & $\{0\}$ & yes & $e^{-t}+\frac{1}{t}$ \\
$\forall m$ & no & hyperplane & yes & $e^{-t}+\frac{1}{t}+\frac{1}{\sqrt{t}}$ \\
& no & $\mathbb{R}^{m}$ & no & $e^{-t}+$ tr. wave \\
\hline$\forall n$ & yes & $\{0\}$ & yes & $e^{-t}+\frac{1}{t^{m / 2}}$ \\
$\forall m$ & no & Uvs codim r & yes & conjecture $:$ \\
& & & & $e^{-t}+\frac{1}{t^{\frac{m}{2}}}+\frac{1}{t^{\frac{r}{(2(n-1))}}}+\frac{1}{t^{\frac{1}{2(n-1)}}}$ \\
& no & submanifold & yes & open problem \\
$\forall n$ & no & $\mathbb{R}^{m}$ & no & open problem \\
$\forall m$ & ns codim r & yes & $e^{-t}+\frac{1}{t^{\frac{m}{2}}}+\frac{1}{t^{\frac{T}{2}}}+\frac{1}{\sqrt{t}}$ \\
$n_{1}=1$ & & & & \\
\hline
\end{tabular}

In this array, by the expression "tr. waves" in the second line we mean "a sum of a finite number of traveling waves", and in the fifth one "a traveling wave". The notation " $\cup$ vs codim $r$ " in the seventh line means that $\mathcal{D}\left(A_{1}, \ldots, A_{m}, B\right)$ is the union of a finite number of vector subspaces $\mathcal{D}_{j}, j=1, \ldots, J$ such that $\operatorname{codim}\left(\mathcal{D}_{j}\right) \geqslant r$ for $j=1, \ldots, J$. The expression "vs codim $\mathrm{r}$ " in the last line means that $\mathcal{D}\left(A_{1}, \ldots, A_{m}, B\right)$ is a vector subspace with codimension $r$.

In the first and second lines we collect the results of Proposition 5. The third, fourth and fifth lines refer to Theorem 4. The sixth line comes from Theorem 1. The seventh line is explained in the next subsection. The eighth and ninth lines summarize the results of Propositions 2, 3 and 4. Finally, the tenth line contains the results of Theorem 2.

\subsection{Open problems and conjectures}

The decomposition of the solutions of (4) when $m \geqslant 2, n \geqslant 3$ are arbitrary is an open problem.

The simplest situation not covered in this article should be the case when the set of degeneracy is a finite union of vectors subspaces of $\mathbb{R}^{m}$. We conjecture that, in that case, the following inequality holds

$$
N_{*, \epsilon}(\omega) \geqslant \operatorname{cdist}(\omega, \mathcal{D})^{2(n-1)} .
$$

The intuition of the exponent $2(n-1)$ comes from the definition

$$
N_{*, \epsilon}(\omega):=\min \left\{\sum_{k=0}^{n-1} \epsilon^{m_{k}}\left|B A(\omega)^{k} x\right|^{2} ; x \in S^{n-1}\right\} .
$$

Indeed, for every $x \in S^{n-1}, \sum_{k=0}^{n-1} \epsilon^{m_{k}}\left|B A(\omega)^{k} x\right|^{2}$ is polynomial of $\omega$ with degree $\leqslant 2(n-1)$. If the conjecture $(67)$ is valid, then Proposition 7 leads to a decomposition for the solutions of (4) when $\mathcal{D}\left(A_{1}, \ldots, A_{m}, B\right)$ is a finite union of 
vectors subspaces of $\mathbb{R}^{m}$. This decomposition is written in the seventh line of the array.

Similarly, when the set of degeneracy is a strict algebraic submanifold of $\mathbb{R}^{m}$ of codimension $r$, we conjecture that any solution of (4) can be decomposed as $w=w_{1}+w_{2}+w_{3}+w_{4}$ where $w_{1}$ and $w_{2}$ satisfy (14) and

$$
\begin{aligned}
& \left\|w_{3}(t)\right\|_{L^{\infty}} \leqslant \frac{C}{t^{\frac{1}{2(n-1)}}} N\left(w_{0}\right), \\
& \left\|w_{4}(t)\right\|_{L^{\infty}} \leqslant \frac{C}{\frac{1}{t^{2(n-1)}}}\|w\|_{L^{1}},
\end{aligned}
$$

where the constant $C$ depends on the parameterization of the algebraic manifold $\mathcal{D}\left(A_{1}, \ldots, A_{m}, B\right)$. However, in this situation, it would not be sufficient to understand the behavior of $N_{*, \epsilon}(\omega)$ since one would also need the analogue of Proposition 7 .

Another open problem is the nature of the non dissipated solutions and the decomposition of the solutions when $\mathcal{D}\left(A_{1}, \ldots, A_{m}, B\right)=\mathbb{R}^{m}$.

\section{Global existence around a constant equilibrium for the nonlinear system}

\subsection{Problem formulation}

In this section, we study non linear systems of balance laws of the form

$$
\frac{\partial w}{\partial t}+\sum_{j=1}^{m} \frac{\partial F_{j}(w)}{\partial x_{j}}=Q(w)
$$

where $m, n \in \mathbb{N}^{*}, w: \mathbb{R} \times \mathbb{R}^{m} \rightarrow \mathbb{R}^{n}, w=w(t, x)$ is the unknown, $F_{j}, Q: \mathbb{R}^{n} \rightarrow$ $\mathbb{R}^{n}$ are smooth functions, and

$$
Q(w):=\left(\begin{array}{c}
0 \\
q(w)
\end{array}\right)
$$

where $0 \in \mathbb{R}^{n_{1}}$ and $q(w) \in \mathbb{R}^{n_{2}}$.

We consider a constant equilibrium $W_{e} \in \mathbb{R}^{n}$ i.e. $Q\left(W_{e}\right)=0$. The aim of this section is to investigate under what conditions the source term may prevent the breakdown of smooth solutions, in a neighborhood of $W_{e}$. This question has been addressed earlier, for instance, in [23], under the following assumptions (H0)-(H2) in order to deal with strictly entropy dissipative symmetrizable systems.

(H0) : The differential $d_{w_{2}} q\left(W_{e}\right)$ is invertible.

(H1) : There exists a strictly convex entropy $\eta=\eta(w)$, defined in a convex compact neighborhood $G$ of $W_{e}$, such that $d_{w}^{2} \eta(w) d_{w} F_{j}(w)$ is symmetric for every $w \in G$ and for every $j \in\{1, \ldots, m\}$.

(H2) : There exists a constant $C_{G}>0$ such that, for every $w \in G$,

$$
\left[d_{w} \eta(w)-d_{w} \eta\left(W_{e}\right)\right] \cdot Q(w) \leqslant-C_{G}|Q(w)|^{2}, \forall w \in G .
$$


Under these conditions, in [23] the existence of global smooth solutions in a neighborhood of $W_{e}$ was proved, when the linearized system around $W_{e}$ satisfies (SK).

We shall also assume that the hypotheses (H0)-(H2) are fulfilled. Moreover, in order to simplify the proof, we will assume the following assumption (H3).

(H3) : There exists $D \in \mathbb{R}^{n_{2} \times n_{2}}$ positive definite such that

$$
Q(w):=\left(\begin{array}{c}
0 \\
-D w_{2}
\end{array}\right)
$$

In subsection 6.2, we make precise Yong's statement in [23] by giving an explicit estimate of the size of the neighborhood of $W_{e}$ in which global existence holds. The possibility of measuring this size explicitly is a key ingredient in the degenerate case, studied in subsection 6.3 , where we show that the (SK) condition on the linearized system around $W_{e}$ is not necessary for global existence around $W_{e}$. More precisely, in subsection 6.3 , we consider a constant degenerate equilibrium $W_{e}$ and we assume the existence of a sequence of non degenerate equilibria $\left(W_{e}^{p}\right)_{p \in \mathbb{N}}$ converging to $W_{e}$ and such that the quantity $N_{*, W_{e}^{p}}$ in $(24)$ measuring the decay rate $(25)$ for the linearized system around $W_{e}^{p}$ converges to zero slowly enough. Then, we prove the existence of global smooth solutions for (68) in a neighborhood of $W_{e}$. Our theorem takes advantage of the contribution of the nonlinearity, when the linearized system is degenerate. Our proof is inspired by Coron's return method for nonlinear control (see [4]). We end this section with an example of application of this theorem.

\subsection{Size of the neighborhood for global existence under (SK)}

In this section, we assume that (SK) holds. More precisely, it is supposed that the following hypothesis is fulfilled.

(H4) : The linearized system around $W_{e}$ satisfies $(\mathrm{SK})$,

$$
\operatorname{Ker}(B) \cap\left\{\text { eigenvectors of } A_{W_{e}}(\omega)\right\}=\{0\}, \forall \omega \in S^{m-1},
$$

where $A_{W_{e}}(\omega):=\sum_{j=1}^{m} \omega_{j} A_{j}\left(W_{e}\right), A_{j}\left(W_{e}\right):=d_{w} F_{j}\left(W_{e}\right)$ and

$$
B:=\left(\begin{array}{cc}
0 & 0 \\
0 & D
\end{array}\right) \text {. }
$$

Thus, using the same notations as in Proposition 1, we deduce that

$$
N_{*, W_{e}}:=\min \left\{\sum_{k=0}^{n-1} \epsilon^{m_{k}}\left|B A_{W_{e}}(\omega)^{k} x\right|^{2} ; x \in S^{n-1}, \omega \in S^{m-1}\right\}>0 .
$$

We now introduce the compensating function

$$
K_{W_{e}}(\omega):=\sum_{k=1}^{n-1} \epsilon^{m_{k}}\left[A_{W_{e}}(\omega)^{* k} B^{*} B A_{W_{e}}(\omega)^{k-1}-A_{W_{e}}(\omega)^{* k-1} B^{*} B A_{W_{e}}(\omega)^{k}\right]
$$

with the notations of Proposition 1 (see the definition of a compensation function in Remark 5). We know that

$$
\frac{B^{*}+B}{2}+\frac{1}{2}\left(K_{W_{e}}(\omega) A_{W_{e}}(\omega)+\left(K_{W_{e}}(\omega) A_{W_{e}}(\omega)\right)^{*}\right) \geqslant \frac{1}{2} N_{*, W_{e}} .
$$


We introduce

$$
\begin{gathered}
C_{W_{e}}:=\max \left\{\left\|K_{W_{e}}(\omega)\right\| ; \omega \in S^{m-1}\right\}, \\
\delta_{W_{e}}:=\frac{1}{2} \min \left\{\frac{1}{C_{W_{e}}}, \frac{N_{*, W_{e}}}{2 C_{W_{e}}^{2}\|D\|^{2}+\|D\| N_{*, W_{e}}}\right\} .
\end{gathered}
$$

Theorem 5 We assume that (HO), (H1), (H2), (H3) and (H4) are fulfilled. Let $s \geqslant[m / 2]+2$ be an integer and $\mathcal{M}>0$ be such that

$$
\|Q\|_{C^{s}(G)}+\sum_{j=1}^{m}\left\|A_{j}\right\|_{C^{s}(G)} \leqslant \mathcal{M} .
$$

Then, there exist $\nu_{p}=\nu_{p}(\eta, G, \mathcal{M})>0$ for $p=1,2,3$ such that, for every $w_{0} \in W_{e}+H^{s}\left(\mathbb{R}^{m}, \mathbb{R}^{n}\right)$ with

$$
\left\|w_{0}-W_{e}\right\|_{H^{s}\left(\mathbb{R}^{m}, \mathbb{R}^{n}\right)}<\nu_{1} N_{*, W_{e}} \min \left\{\delta_{W_{e}}, \nu_{2}\right\},
$$

the system (68) has a unique global solution

$$
w \in C^{0}\left([0,+\infty), W_{e}+H^{s}\left(\mathbb{R}^{m}, \mathbb{R}^{n}\right)\right)
$$

satisfying, for every $T>0$,

$$
\left\|w(T)-W_{e}\right\|_{H^{s}}^{2}+\int_{0}^{T}\left\|w_{2}\right\|_{H^{s}}^{2}+\frac{\delta_{W_{e}} N_{*, W_{e}}}{2} \int_{0}^{T}\left\|\nabla_{x} w\right\|_{H^{s-1}}^{2} \leqslant \nu_{3}\left\|w_{0}-W_{e}\right\|_{H^{s}}^{2} .
$$

Proof of Theorem 5: Thanks to the assumption (H3), we have $W_{e 2}=0$. We proceed in several steps.

Step 1: Let $w_{0} \in W_{e}+H^{s}\left(\mathbb{R}^{m}, \mathbb{R}^{n}\right)$. From the local in time existence theory of $H^{s}$ solutions, we know that there exists $T>0$ depending only on $\left\|w_{0}-W_{e}\right\|_{H^{s}}$ such that (68), with initial value $w_{0}$, has a unique solution

$$
w \in C^{0}\left([0, T], W_{e}+H^{s}\left(\mathbb{R}^{m}, \mathbb{R}^{n}\right)\right) .
$$

As long as (75) holds, one has

$$
\left\|w(T)-W_{e}\right\|_{H^{s}\left(\mathbb{R}^{m}, \mathbb{R}^{n}\right)} \leqslant \sqrt{\nu_{3}}\left\|w_{0}-W_{e}\right\|_{H^{s}} .
$$

Since the right hand side of this estimate does not depend on $T$, the solution can be continued up to $T=+\infty$. Thus, to prove the theorem, we only need to justify the bound (75).

Step 2: Let us now prove (75). Arguing as in [23, pp 255-259] we get the existence of $\mathcal{C}_{p}=\mathcal{C}_{p}(\eta, G, \mathcal{M})>0$, for $p=1,2$ such that

$$
\begin{aligned}
& \left\|w(T)-W_{e}\right\|_{H^{s}}^{2}+\int_{0}^{T}\left\|w_{2}(t)\right\|_{H^{s}}^{2} d t \\
\leqslant & \mathcal{C}_{1}\left\|w_{0}-W_{e}\right\|_{H^{s}}^{2}+\mathcal{C}_{2} N_{s}(T) \int_{0}^{T}\left(\left\|\nabla_{x} w(t)\right\|_{H^{s-1}}^{2}+\left\|w_{2}(t)\right\|_{H^{s-1}}^{2}\right) d t
\end{aligned}
$$

where

$$
N_{s}(T):=\sup _{0 \leqslant t \leqslant T}\left\|w(t)-W_{e}\right\|_{H^{s}}
$$


In order to control the term $\int_{0}^{T}\left\|\nabla_{x} w(t)\right\|_{H^{s-1}}^{2} d t$, we use the linearized system around $W_{e}$. Here we develop the arguments in [23] making them more precise. We have

$$
\frac{\partial w}{\partial t}+\sum_{j=1}^{m} A_{j}\left(W_{e}\right) \frac{\partial w}{\partial x_{j}}=Q(w)+h_{W_{e}}
$$

where

$$
h_{W_{e}}:=\sum_{j=1}^{m}\left[A_{j}\left(W_{e}\right)-A_{j}(w)\right] \frac{\partial w}{\partial x_{j}} .
$$

Applying the Fourier transform, we get

$$
\frac{\partial \hat{w}}{\partial t}+i A_{W_{e}}(\xi) \hat{w}=\widehat{Q(w)}+\hat{h}_{W_{e}}
$$

We compute the imaginary part of the scalar product in $\mathbb{C}^{n}$ of equation (77) with $K_{W_{e}}(\omega) \hat{w}(t, \xi)$ (where $\left.\xi=|\xi| \omega\right)$. Using the skew symmetry of $K_{W_{e}}(\omega)$, we get

$$
\Im\left(\left\langle K_{W_{e}}(\omega) \hat{w}, \frac{\partial \hat{w}}{\partial t}\right\rangle\right)+|\xi|\left\langle\hat{w}, K_{W_{e}}(\omega) A_{W_{e}}(\omega) \hat{w}\right\rangle=\Im\left\langle K_{W_{e}}(\omega) \hat{w}, \widehat{Q(w)}+\hat{h}_{W_{e}}\right\rangle .
$$

Thanks to (71), (72) and Cauchy-Schwarz inequality, we get

$$
\begin{aligned}
|\xi|\left(\frac{N_{*, W_{e}}}{2}|\hat{w}|^{2}-\|D\|\left|\hat{w}_{2}\right|^{2}\right) \leqslant & -\Im\left\langle K_{W_{e}}(\omega) \hat{w}, \frac{\partial \hat{w}}{\partial t}\right\rangle+C_{W_{e}}|\hat{w}|\left(|\widehat{Q(w)}|+\left|\hat{h}_{W_{e}}\right|\right) \\
\leqslant & -\Im\left\langle K_{W_{e}}(\omega) \hat{w}, \frac{\partial \hat{w}}{\partial t}\right\rangle+\frac{N_{*}, W_{e}|\xi|}{4}|\hat{w}|^{2} \\
& +\frac{2 C_{W_{e}}^{2}}{N_{*, W_{e}|\xi|}}\left(|\widehat{Q(w)}|^{2}+\left|\hat{h}_{W_{e}}\right|^{2}\right) .
\end{aligned}
$$

Multiplying this inequality by $|\xi|^{2 k-1}$, integrating over $(0, T)_{t} \times \mathbb{R}_{\xi}^{m}$, and summing over $k \in\{1, \ldots, s\}$, we get

$$
\begin{aligned}
\frac{N_{*, W_{e}}}{4} \int_{0}^{T}\left\|\nabla_{x} w\right\|_{H^{s-1}}^{2} d t \leqslant & C_{W_{e}}\left[\left\|w(T)-W_{e}\right\|_{H^{s}}^{2}+\left\|w_{0}-W_{e}\right\|_{H^{s}}^{2}\right] \\
& +\int_{0}^{T}\|D\|\left\|w_{2}\right\|_{H^{s}}^{2} d t \\
& +\frac{2 C_{W_{e}}^{2}}{N_{*, W_{e}}} \int_{0}^{T}\|Q(w)\|_{H^{s-1}}^{2}+\left\|h_{W_{e}}\right\|_{H^{s-1}}^{2} d t .
\end{aligned}
$$

Indeed, we have

$$
\begin{aligned}
& \sum_{k=1}^{s} \int_{0}^{T} \int_{\mathbb{R}^{m}}|\xi|^{2 k-1} \Im\left\langle K_{W_{e}}(\omega) \hat{w}, \frac{\partial \hat{w}}{\partial t}\right\rangle d \xi d t \\
& =\sum_{k=1}^{s} \int_{\mathbb{R}^{m}}|\xi|^{2 k-1}\left(\left\langle K_{W_{e}}(\omega) \hat{w}(T), \hat{w}(T)\right\rangle-\left\langle K_{W_{e}}(\omega) \hat{w}(0), \hat{w}(0)\right\rangle\right) d \xi \\
& \leqslant C_{W_{e}} \sum_{k=1}^{s} \int_{\mathbb{R}^{m}}|\xi|^{2 k-1}\left(|\hat{w}(T)|^{2}+\left|\hat{w}_{0}\right|^{2}\right) d \xi \\
& \leqslant C_{W_{e}}\left(\left\|w(T)-W_{e}\right\|_{H^{s}}^{2}+\left\|w_{0}-W_{e}\right\|_{H^{s}}^{2}\right) .
\end{aligned}
$$

Let $\widetilde{\mathcal{C}}_{\mathcal{M}}>0$ be such that, for every $t \in(0, T)$,

$$
\left\|h_{W_{e}}(t)\right\|_{H^{s-1}} \leqslant \sum_{j=1}^{m}\left|A_{j}\right|_{C^{s-1}(G)} N_{s}(T)\left\|\frac{\partial w}{\partial x_{j}}\right\|_{H^{s-1}} \leqslant \widetilde{\mathcal{C}}_{\mathcal{M}} N_{s}(T)\left\|\nabla_{x} w(t)\right\|_{H^{s-1}}
$$

We get

$$
\begin{aligned}
\frac{N_{*, W_{e}}}{4} \int_{0}^{T}\left\|\nabla_{x} w\right\|_{H^{s-1}}^{2} d t & \leqslant C_{W_{e}}\left[\left\|w(T)-W_{e}\right\|_{H^{s}}^{2}+\left\|w_{0}-W_{e}\right\|_{H^{s}}^{2}\right] \\
& +\left(\|D\|+\frac{2 C_{W_{e}}^{2}\|D\|^{2}}{N_{*, \epsilon}}\right) \int_{0}^{T}\left\|w_{2}\right\|_{H^{s}}^{2} d t \\
& +\frac{2 C_{W_{e}}^{2} \widetilde{\mathcal{C}}_{\mathcal{M}}^{2}}{N_{*, W_{e}}} N_{s}(T)^{2} \int_{0}^{T}\left\|\nabla_{x} w\right\|_{H^{s-1}}^{2} d t .
\end{aligned}
$$


Thus multiplying (79) by $\delta_{W_{e}}$ and adding (76), we get

$$
\leqslant \begin{aligned}
& \frac{1}{2}\left\|w(T)-W_{e}\right\|_{H^{s}}^{2}+\left(\frac{1}{2}-\mathcal{C}_{2} N_{s}(T)\right) \int_{0}^{T}\left\|w_{2}\right\|_{H^{s}}^{2}+\frac{\delta_{W_{e}} N_{*, W_{e}}}{4} \int_{0}^{T}\left\|\nabla_{x} w\right\|_{H^{s-1}}^{2} \\
& \left(\mathcal{C}_{1}+\frac{1}{2}\right)\left\|w_{0}-W_{e}\right\|_{H^{s}}^{2}+ \\
& \left(\mathcal{C}_{2} N_{s}(T)+2 \delta_{W_{e}} \widetilde{\mathcal{C}}_{\mathcal{M}^{2}}^{2} N_{s}(T)^{2} \frac{C_{W_{e}}^{2}}{N_{*}, W_{e}}\right) \int_{0}^{T}\left\|\nabla_{x} w\right\|_{H^{s-1}}^{2} .
\end{aligned}
$$

Indeed, the definition of $\delta_{W_{e}}$ (see (73)) implies

$$
\delta_{W_{e}}\left(\|D\|^{2}+\frac{2 C_{W_{e}^{2}} \mathcal{C}_{\mathcal{M}}}{N_{*, \epsilon}}\right) \leqslant \frac{1}{2}
$$

and

$$
\delta_{W_{e}} C_{W_{e}} \leqslant \frac{1}{2} .
$$

So, as long as $N_{s}(T)$ is small enough so that $w(t,.) \in G$ on $(0, T), \mathcal{C}_{2} N_{s}(T)<1 / 4$ and

$$
\mathcal{C}_{2} N_{s}(T)+2 \delta_{W_{e}} \widetilde{\mathcal{C}}_{\mathcal{M}}^{2} N_{s}(T)^{2} \frac{C_{W_{e}}^{2}}{N_{*, W_{e}}} \leqslant \frac{\delta_{W_{e}} N_{*, W_{e}}}{8}
$$

we have (75) with $\nu_{3}:=4\left(\mathcal{C}_{1}+1\right)$ and

$$
\left\|w(T)-W_{e}\right\|_{H^{s}} \leqslant 2 \sqrt{\mathcal{C}_{1}+1}\left\|w_{0}-W_{e}\right\|_{H^{s}} .
$$

The inequality (82) holds in particular when

$$
\mathcal{C}_{2} N_{s}(T) \leqslant \frac{\delta_{W_{e}} N_{*, W_{e}}}{16} \quad \text { and } \quad 2 \delta_{W_{e}} \widetilde{\mathcal{C}}_{\mathcal{M}}^{2} N_{s}(T)^{2} \frac{C_{W_{e}}^{2}}{N_{*}, W_{e}} \leqslant \frac{\delta_{W_{e}} N_{*}, W_{e}}{16},
$$

i.e. when

$$
N_{s}(T) \leqslant N_{*, W_{e}} \min \left\{\frac{\delta_{W_{e}}}{16 \mathcal{C}_{2}}, \frac{1}{4 \sqrt{2} \widetilde{\mathcal{C}}_{\mathcal{M}} C_{W_{e}}}\right\} .
$$

The inequality (83) justifies that (84) holds as soon as

$$
\left\|w_{0}-W_{e}\right\|_{H^{s}}<\frac{1}{2 \sqrt{\mathcal{C}_{1}+1}} N_{*, W_{e}} \min \left\{\frac{\delta_{W_{e}}}{16 \mathcal{C}_{2}}, \frac{1}{4 \sqrt{2} \widetilde{\mathcal{C}}_{\mathcal{M}} C_{W_{e}}}\right\} .
$$

We have proved Theorem 5 with

$$
\nu_{1}:=\frac{1}{32 \mathcal{C}_{2} \sqrt{\mathcal{C}_{1}+1}}, \quad \nu_{2}:=\frac{4 \mathcal{C}_{2}}{\sqrt{2} \tilde{\mathcal{C}}_{\mathcal{M}} C_{W_{e}}}, \quad \nu_{3}:=4\left(\mathcal{C}_{1}+1\right) .
$$

Remark 15 Thanks to the careful study of the linear hyperbolic systems in the previous sections, one sees that Yong's strategy [23] we have pursued here cannot be applied directly to the case where the linearized system around the equilibrium $W_{e}$ does not satisfy (SK). For example, if the linearized system around $W_{e}$ is such that

$$
N_{*, W_{e}}(\omega) \geqslant \operatorname{cdist}(\omega, \mathcal{D})^{2},
$$

we cannot get a control on the whole term $\int_{0}^{T}\left\|\nabla_{x} w(t)\right\|_{H^{s-1}}^{2} d t$ but only on part of the gradient. This corresponds to the situation where some of the space directions are missing in the first order derivatives in the linearized system. In that case, the PDE does not have any structure on those missing variables which are only unstructured parameters, the gain of time-space estimates being impossible on those directions. 


\subsection{Global existence without (SK)}

In this subsection, we assume the following hypothesis.

(H5) The linearized system around $W_{e}$ does not satisfy (SK), but there exists a sequence of constant equilibria $\left(W_{e}^{p}\right)_{p \in \mathbb{N}} \subset G$ such that

- $W_{e}^{p} \rightarrow W_{e}$ when $p \rightarrow+\infty$,

- for every $p \in \mathbb{N}$, the linearized system around $W_{e}^{p}$ satisfies $(\mathrm{SK})$.

Theorem 6 We assume that (HO)- (H3) and (H5) are fulfilled. Let $s \geqslant$ $[m / 2]+2$ be an integer and $\mathcal{M}>0$ be such that (74) holds. There exist $\nu_{k}=$ $\nu_{k}(\eta, G, \mathcal{M})>0$ for $k=0,1,2,3$ such that, if

$$
N_{*}\left(W_{e}^{p}\right)>\nu_{0} C_{W_{e}^{p}}\left|W_{e}^{p}-W_{e}\right| \text {, for p large enough, }
$$

then, there exists $p \in \mathbb{N}^{*}$ such that for every $w_{0} \in W_{e}+H^{s}\left(\mathbb{R}^{m}, \mathbb{R}^{n}\right)$ with

$$
\left\|w_{0}-W_{e}\right\|_{H^{s}\left(\mathbb{R}^{m}, \mathbb{R}^{n}\right)}<\nu_{1} N_{*, W_{e}^{p}} \min \left\{\delta_{W_{e}^{p}}, \nu_{2}\right\}
$$

the system (68) has a unique global solution $w \in C^{0}\left([0,+\infty), W_{e}+H^{s}\left(\mathbb{R}^{m}, \mathbb{R}^{n}\right)\right)$ satisfying, for every $T>0$,

$\left\|w(T)-W_{e}\right\|_{H^{s}}^{2}+\int_{0}^{T}\left\|w_{2}\right\|_{H^{s}}^{2}+\frac{\delta_{W_{e}^{p}} N_{*, W_{e}^{p}}}{2} \int_{0}^{T}\left\|\nabla_{x} w\right\|_{H^{s-1}}^{2} \leqslant \nu_{3}\left\|w_{0}-W_{e}\right\|_{H^{s}}^{2}$.

Remark 16 Obviously (H5) implies that $N_{*, W_{e}^{p}} \rightarrow 0$ when $p \rightarrow+\infty$. The assumption (85) only says that this convergence is not too fast.

Proof of Theorem 6 : This proof is an adaptation of the proof of Theorem 5 and the same notations will be used.

As in the proof of Theorem 5 , there exists $\mathcal{C}_{1}=\mathcal{C}_{1}(\eta, G)>0, \mathcal{C}_{2}=$ $\mathcal{C}_{2}\left(\eta, G, A_{1}, \ldots, A_{m}\right)>0$ such that (76) holds.

In order to control $\int_{0}^{T}\left\|\nabla_{x} w(t)\right\|_{H^{s-1}}^{2} d t$, we use the linearized system around $W_{e}^{p}$ for $p$ large enough writing

$$
\frac{\partial w}{\partial t}+\sum_{j=1}^{m} A_{j}\left(W_{e}^{p}\right) \frac{\partial w}{\partial x_{j}}=Q(w)+h_{W_{e}^{p}}
$$

Applying the Fourier transform, we get

$$
\frac{\partial \hat{w}}{\partial t}+i A_{W_{e}^{p}}(\xi) \hat{w}=\widehat{Q(w)}+\hat{h}_{W_{e}^{p}}
$$

We compute the imaginary part of the scalar product of this equation with $K_{W_{e}^{p}}(\omega) \hat{w}(t, \xi)$. Performing the same analysis as in the proof of Theorem 5 , we get

$$
\begin{aligned}
\frac{N_{*, W_{e}^{p}}^{p}}{4} \int_{0}^{T}\left\|\nabla_{x} w\right\|_{H^{s-1}}^{2} d t \leqslant & C_{W_{e}^{p}}\left[\left\|w(T)-W_{e}\right\|_{H^{s}}^{2}+\left\|w_{0}-W_{e}\right\|_{H^{s}}^{2}\right] \\
& +\left(\|D\|+\frac{2 C_{W_{e}^{p}}^{2}\|D\|^{2}}{N_{*, W_{e}^{p}}}\right) \int_{0}^{T}\left\|w_{2}(t)\right\|_{H^{s}}^{2} d t \\
& +\frac{2 C_{W_{e}^{p}}^{2}}{N_{*, W_{e}^{p}}} \int_{0}^{T}\left\|h_{W_{e}^{p}}(t)\right\|_{H^{s-1}}^{2} d t .
\end{aligned}
$$


Moreover, there exists $\widetilde{\mathcal{C}}_{\mathcal{M}}>0$ such that for every $t \in(0, T)$,

$$
\begin{aligned}
\left\|h_{W_{e}^{p}}(t)\right\|_{H^{s-1}} & =\left\|h_{W_{e}}(t)+\sum_{j=1}^{m}\left[A_{j}\left(W_{e}\right)-A_{j}\left(W_{e}^{p}\right)\right] \frac{\partial w}{\partial x_{j}}(t)\right\|_{H^{s-1}} \\
& \leqslant \widetilde{\mathcal{C}}_{\mathcal{M}}\left[N_{s}(T)+\left|W_{e}-W_{e}^{p}\right|\right]\left\|\nabla_{x} w(t)\right\|_{H^{s-1}}
\end{aligned}
$$

Thus,

$$
\begin{aligned}
\frac{N_{*, W_{e}^{p}}^{p}}{4} \int_{0}^{T}\left\|\nabla_{x} w\right\|_{H^{s-1}}^{2} d t \leqslant & C_{W_{e}^{p}}\left[\left\|w(T)-W_{e}\right\|_{H^{s}}^{2}+\left\|w_{0}-W_{e}\right\|_{H^{s}}^{2}\right] \\
& +\left(\|D\|+\frac{2 C_{W_{e}^{p}}^{2}\|D\|^{2}}{N_{*, W_{e}^{p}}}\right) \int_{0}^{T}\left\|w_{2}(t)\right\|_{H^{s}}^{2} \\
& +\frac{4 C_{W_{e}^{p}}^{2} \widetilde{\mathcal{C}}_{\mathcal{M}}^{2}}{N_{*, W_{e}^{p}}^{p}}\left(N_{s}(T)^{2}+\left|W_{e}-W_{e}^{p}\right|^{2}\right) \int_{0}^{T}\left\|\nabla_{x} w\right\|_{H^{s-1}}^{2} d t
\end{aligned}
$$

Multiplying (90) by $\delta_{W_{e}^{p}}$ and adding (76), as long as $\mathcal{C}_{2} N_{s}(T)<1 / 4$, we get

$$
\begin{aligned}
& \frac{1}{2}\left\|w(T)-W_{e}\right\|_{H^{s}}^{2}+\frac{1}{4} \int_{0}^{T}\left\|w_{2}\right\|_{H^{s}}^{2}+\frac{\delta_{W_{e}^{p} N_{*, W_{e}^{p}}}}{4} \int_{0}^{T}\left\|\nabla_{x} w\right\|_{H^{s-1}}^{2} \\
& \leqslant\left(\mathcal{C}_{1}+\frac{1}{2}\right)\left\|w_{0}-W_{e}\right\|_{H^{s}}^{2} \\
& +\left(\mathcal{C}_{2} N_{s}(T)+4 \delta_{W_{e}^{p}} \widetilde{\mathcal{C}}_{\mathcal{M}}^{2}\left[N_{s}(T)^{2}+\left|W_{e}-W_{e}^{p}\right|^{2}\right] \frac{C_{W_{e}^{p}}^{2}}{N_{*, W_{e}^{p}}}\right) \int_{0}^{T}\left\|\nabla_{x} w\right\|_{H^{s-1}}^{2} d t .
\end{aligned}
$$

So, as long as

$$
\mathcal{C}_{2} N_{s}(T)+4 \delta_{W_{e}^{p}} \widetilde{\mathcal{C}}_{\mathcal{M}}^{2}\left[N_{s}(T)^{2}+\left|W_{e}-W_{e}^{p}\right|^{2}\right] \frac{C_{W_{e}^{p}}^{2}}{N_{*, W_{e}^{p}}} \leqslant \frac{\delta_{W_{e}^{p}} N_{*, W_{e}^{p}}}{8},
$$

we have (87) with $\nu_{3}:=4\left(\mathcal{C}_{1}+1\right)$. The inequality (91) holds in particular when

$$
\begin{gathered}
\mathcal{C}_{2} N_{s}(T) \leqslant \frac{\delta_{W_{e}^{p} N_{*, W_{e}^{p}}}}{24}, \\
4 \delta_{W_{e}^{p}} \widetilde{\mathcal{C}}_{\mathcal{M}}^{2} \frac{C_{W_{e}^{p}}^{2}}{N_{*, W_{e}^{p}}} N_{s}(T)^{2} \leqslant \frac{\delta_{W_{e}^{p}} N_{*, W_{e}^{p}}}{24}, \\
4 \delta_{W_{e}^{p}} \widetilde{\mathcal{C}}_{\mathcal{M}}^{2} \frac{C_{W_{e}^{p}}^{2}}{N_{*, W_{e}^{p}}}\left|W_{e}-W_{e}^{p}\right|^{2} \leqslant \frac{\delta_{W_{e}^{p}} N_{*, W_{e}^{p}}}{24},
\end{gathered}
$$

i.e. when

$$
N_{s}(T) \leqslant \min \left\{\frac{\delta_{W_{e}^{p} N_{*, W_{e}^{p}}}}{24 \mathcal{C}_{2}}, \frac{N_{*, W_{e}^{p}}}{\sqrt{96} C_{W_{e}^{p}} \widetilde{\mathcal{C}}_{\mathcal{M}}}\right\}
$$

and

$$
\left|W_{e}-W_{e}^{p}\right| \leqslant \frac{N_{*, W_{e}^{p}}}{\sqrt{96} C_{W_{e}^{p}} \widetilde{\mathcal{C}}_{\mathcal{M}}} .
$$

The inequality (87) proves that (92) holds when

$$
\left\|w_{0}-W_{e}\right\|_{H^{s}} \leqslant \frac{1}{2 \sqrt{\mathcal{C}_{1}+1}} \min \left\{\frac{\delta_{W_{e}^{p}} N_{*, W_{e}^{p}}}{24 \mathcal{C}_{2}}, \frac{N_{*, W_{e}^{p}}}{\sqrt{96} C_{W_{e}^{p}} \widetilde{\mathcal{C}}_{\mathcal{M}}}\right\} .
$$

We have proved Theorem 6 with

$$
\nu_{0}:=\sqrt{96} \widetilde{\mathcal{C}}_{\mathcal{M}}, \nu_{1}:=\frac{1}{48 \mathcal{C}_{2} \sqrt{\mathcal{C}_{1}+1}}, \nu_{2}:=\frac{\sqrt{24} \mathcal{C}_{2}}{2 C_{W_{e}^{p}} \widetilde{\mathcal{C}}_{\mathcal{M}}}, \nu_{3}:=4\left(\mathcal{C}_{1}+1\right) . \square
$$

Of course, the assumption (H6) may be difficult to check on a given system of conservation laws. But it gives an easy way to build examples of non linear 
systems of conservation laws with global smooth solutions in a neighborhood of a degenerate constant equilibrium.

Example of application of Theorem 6 : Let us consider (68) with $m=1, n=2$,

$$
F(w)=\left(\begin{array}{c}
F^{(1)}(w) \\
F^{(2)}(w)
\end{array}\right), \quad Q(w)=\left(\begin{array}{c}
0 \\
w_{2}
\end{array}\right), \quad W_{e}=\left(\begin{array}{c}
0 \\
0
\end{array}\right), \quad W_{e}^{p}=\left(\begin{array}{c}
a_{p} \\
0
\end{array}\right)
$$

where $a_{p} \neq 0$ and $a_{p} \rightarrow 0$. We assume

$$
\frac{\partial F^{(2)}}{\partial w_{1}}\left(W_{e}\right)=0
$$

$$
\frac{\partial F^{(1)}}{\partial w_{2}}(w)=\frac{\partial F^{(2)}}{\partial w_{1}}(w), \forall w \in G
$$

where $G$ is a neighborhood of $W_{e}$ in $\mathbb{R}^{2}$,

- there exists $M>0$ such that

$$
\left|\frac{\partial F^{(2)}}{\partial w_{2}}\left(W_{e}^{p}\right)\right| \leqslant M\left|\frac{\partial F^{(1)}}{\partial w_{2}}\left(W_{e}^{p}\right)\right|, \forall p \in \mathbb{N} .
$$

The assumption (H0) is fulfilled because $q(w)=-w_{2}$ thus $d_{w_{2}} q(w)=-1$ is invertible. The assumption (H1) holds with $\eta(w):=|w|^{2}$ because $d_{w} F(w)$ is symmetric thanks to the assumption (93). The assumption (H2) is fulfilled with $C_{G}=1$. It is clear that (H3) holds.

By definition, $N_{*, W}$ is the smallest eigenvalue of the non negative matrix

$$
\left(\begin{array}{cc}
\epsilon^{m_{1}}\left(\frac{\partial F^{(2)}}{\partial w_{1}}(W)\right)^{2} & \epsilon^{m_{1}} \frac{\partial F^{(2)}}{\partial w_{1}}(W) \frac{\partial F^{(2)}}{\partial w_{2}}(W) \\
\epsilon^{m_{1}} \frac{\partial F^{(2)}}{\partial w_{1}}(W) \frac{\partial F^{(2)}}{\partial w_{2}}(W) & 1+\epsilon^{m_{1}}\left(\frac{\partial F^{(2)}}{\partial w_{2}}(W)\right)^{2}
\end{array}\right),
$$

thus $N_{*, W_{e}}=0$, i.e. (SK) is not satisfied for the linearized system around $W_{e}$ and, for $\epsilon>0$ small enough, we have

$$
N_{*, W_{e}^{p}} \geqslant \frac{1}{2} \epsilon^{m_{1}}\left|\frac{\partial F^{(2)}}{\partial w_{1}}\left(W_{e}^{p}\right)\right|^{2} .
$$

By definition of $C_{W_{e}^{p}}$ and thanks to (94), we have

$$
C_{W_{e}^{p}} \leqslant 2 \epsilon^{m_{1}}(1+M)\left|\frac{\partial F^{(2)}}{\partial w_{1}}\left(W_{e}^{p}\right)\right|
$$

Thus, (85) holds in particular when

$$
\left|\frac{\partial F^{(1)}}{\partial w_{2}}\left(W_{e}^{p}\right)\right|>4 \nu_{0}(1+M)\left|W_{e}^{p}-W_{e}\right|
$$

which is valid, in particular, when

$$
\left|\frac{\partial^{2} F^{(1)}}{\partial w_{2} \partial w_{1}}\left(W_{e}\right)\right|
$$

is large enough. At this point, it is clear that the assumption (85) takes advantage of the nonlinearity. 
Remark 17 Finally, let us notice that Theorem 6 does not apply to the isentropic Euler equation with damping,

$\frac{\partial w}{\partial t}+\frac{\partial F(w)}{\partial x}=\left(\begin{array}{c}0 \\ -w_{2}\end{array}\right), F(w)=\left(\begin{array}{c}-w_{2} \\ f\left(w_{1}\right)\end{array}\right), W_{e}=\left(\begin{array}{l}0 \\ 0\end{array}\right), W_{e}^{p}=\left(\begin{array}{c}a_{p} \\ 0\end{array}\right)$,

where $a_{p} \neq 0$ and $a_{p} \rightarrow 0$ when $p \rightarrow+\infty$. Indeed, easy calculations show that the non validity of (SK) for the linearized system around $W_{e}$ is equivalent to $f^{\prime}(0)=0$ and the assumption (85) is equivalent to

$$
\left|f^{\prime}\left(a_{p}\right)\right|^{2}>\tilde{\nu}_{0}\left|a_{p}\right|, \forall p \in \mathbb{N}^{*} .
$$

This last inequality is impossible when $f$ is smooth. Therefore, the existence of global smooth solutions in a neighborhood of zero, for this equation, when $f^{\prime}(0)=0$ stays an open problem.

\section{References}

[1] D. Aregba-Driollet and R. Natalini. Discrete kinetic schemes for multidimensional systems of conservation laws. SIAM J. Numer. Anal., 37:19732004, 2000.

[2] S. Bianchini, B. Hanouzet, and R. Natalini. Asymptotic behavior of smooth solutions for partially dissipative hyperbolic systems with a convex entropy. IAC Report (in press in Communications in Pure and Applied Mathematics), 79, 2005 .

[3] F. Bouchut. Construction of bgk models with a family of kinetic entropies for a given system of conservation laws. J. Statist. Phys., 95:113-170, 1999.

[4] J.-M. Coron. Control and nonlinearity, volume 136 of Mathematical Surveys and Monographs. American Mathematical Society, 2007.

[5] J.-F. Coulombel and T. Goudon. The strong relaxation limit of the multidimensional isothermal Euler equations. Trans. Am. Math. Soc., 359(2):637648, 2007.

[6] C.M. Dafermos. A system of hyperbolic conservation laws with frictional damping. Z. Angew. Math. Phys., no. Special Issue, S294-S307, Theoretical, experimental and numerical contributions to the mecanics of fluids and solids, 46, 1995.

[7] C.M. Dafermos. Hyperbolic conservation laws in continuum physics. Springer, Berlin, 2000.

[8] B. Hanouzet and P. Huynh. Approximation par relaxation d un système de Maxwell non linéaire. C.R. Acad. Sci. Paris, Sér. I,Math., 330:193-198, 2000 .

[9] B. Hanouzet and R. Natalini. Global existence of smooth solutions for partially dissipative hyperbolic systems with convex entropy. Arch. Ration. Mech. Anal., 169:89-117, 2003. 
[10] A. Haraux and E. Zuazua. Decay estimates for some semilinear damped hyperbolic problems. Arch. Rational Mech. Anal., 100(2):191-206, 1988.

[11] L. Hörmander. Hypoelliptic second order differential equations. Acta Math., 119:147-171, 1967.

[12] T. Kato. Perturbation theory for linear operators. Classics in Mathematics. Springer-Verlag, Berlin, 1995. Reprint of the 1980 edition.

[13] L. Hsiao and T.-P. Liu. Convergence to nonlinear diffusion waves for solutions of a system of hyperbolic conservation laws with damping. Comm. Math. Physics, 143:599-605, 1992.

[14] T.-T. Li. Global classical solutions for quasilinear hyperbolic systems. Masson, Paris, 1994.

[15] A. Majda. Compressible fluid flow and systems of conservation laws in several space variable. Springer, New York, 1984.

[16] R. Natalini. Recent results on hyperbolic relaxation problems. Analysis of systems of conservation laws (Aachen, 1997), Chapman and Hall/CRC, Boca Raton,FL, pages 128-198, 1999.

[17] T. Nishida. Nonlinear hyperbolic equations and related topics in fluid dynamics. Département de mathématiques, Université Paris Sud, Orsay, Prépublications mathématiques d Orsay, 78-02, 1978.

[18] R. Orive and E. Zuazua. Long-time behavior of solutions to a nonlinear hyperbolic relaxation system. J. Differential Equations, 228(1):17-38, 2006.

[19] D. Serre. Systèmes de lois de conservation, tome 1. Diderot editeur, Arts et Sciences, Paris, New-York, Amsterdam, 1996.

[20] Y. Shizuta and S. Kawashima. Systems of equations of hyperbolic-parabolic type with application to the discrete boltzmann equation. Hokkaido Math. $J, 14: 249-275,1985$.

[21] E. Trelat. Contrôle optimal : théorie et applications. Vuilbert, collection Mathématiques Concrètes, 2005.

[22] C. Villani. Hypocoercive diffusion operators. In International Congress of Mathematicians. Vol. III, pages 473-498. Eur. Math. Soc., Zürich, 2006.

[23] W.A. Yong. Entropy and global existence for hyperbolic balance laws. Arch. Ration. Mech. Anal., 172:247-266, 2004.

[24] Y. Zeng. Gas dynamics in thermal nonequilibrium and general hyperbolic systems with relaxation. Arch. Ration. Mech. Anal., 150(3):225-279, 1999.

[25] E. Zuazua. Propagation, observation, and control of waves approximated by finite difference methods. SIAM Rev., 47(2):197-243 (electronic), 2005. 